Article

\title{
Experimental Modeling of Silicate and Carbonate Sulfidation under Lithospheric Mantle P,T-Parameters
}

\author{
Evgeniy Zdrokov ${ }^{1,2}$, Ivan Novoselov ${ }^{1,2}$, Yuliya Bataleva ${ }^{1, *}$, Yuri Borzdov ${ }^{1}$ and \\ Yuri Palyanov $1,2, *$ \\ 1 Sobolev Institute of Geology and Mineralogy, Siberian Branch of Russian Academy of Sciences, \\ Koptyug ave 3, 630090 Novosibirsk, Russia \\ 2 Department of Geology and Geophysics, Novosibirsk State University, Pirogova str 2, \\ 630090 Novosibirsk, Russia \\ * Correspondence: bataleva@igm.nsc.ru (Y.B.); palyanov@igm.nsc.ru (Y.P.); Tel.: +7-383-330-75-01 (Y.P.)
}

Received: 16 June 2019; Accepted: 9 July 2019; Published: 11 July 2019

\begin{abstract}
Interactions of mantle silicates with subducted carbonates, sulfides, and sulfur-rich fluids are experimentally simulated in the olivine-ankerite-sulfur and olivine-ankerite-pyrite systems using a multi-anvil high-pressure split-sphere apparatus at $6.3 \mathrm{GPa}$ and range of $1050-1550{ }^{\circ} \mathrm{C}$. Recrystallization of Fe,Ni-bearing olivine and ankerite in a sulfur melt was found to be accompanied by sulfidation of olivine and carbonate, involving partial extraction of metals, carbon, and oxygen into the melt, followed by the formation of pyrite ( \pm pyrrhotite), diopside, and Fe-free carbonates. The main features of metasomatic alteration of Fe,Ni-olivine by a reduced sulfur fluid include: (i) a zonal structure of crystals (Fe-rich core, $\mathrm{Mg}$-rich rim); (ii) inclusions of pyrite and pyrrhotite in olivine; (iii) certain Raman spectral characteristics of olivine. At $\mathrm{T}>1350{ }^{\circ} \mathrm{C}$, two immiscible melts, a predominantly sulfur melt with dissolved components (or a Fe-Ni-S-O melt) and a predominantly carbonate one, are generated. The redox interaction of these melts leads to the formation of metastable graphite $\left(1350-1550^{\circ} \mathrm{C}\right)$ and diamond growth $\left(1550{ }^{\circ} \mathrm{C}\right)$. The studied olivine-ankerite-sulfur and olivine-ankerite-pyrite interactions may be considered as the basis for simulation of metasomatic processes accompanied by the formation of mantle sulfides during subduction of crustal material to the silicate mantle.
\end{abstract}

Keywords: carbonate; sulfur; sulfide; olivine; sulfidation; mantle metasomatism; subduction; redox interaction; high pressure experiment; lithospheric mantle; diamond

\section{Introduction}

Carbon and sulfur are some of the most common volatile components involved in mantle magmatic and metasomatic processes. As elements with variable valency, they can affect directly the redox evolution of mantle rocks, melts, and fluids. According to the modern data, subduction processes play a key role in the global carbon and sulfur cycles [1-6]. Subduction enables transport of crustal carbon-bearing material (carbonates and hydrocarbons) and sulfur (sulfides and sulfates) minerals to the mantle depths. Several studies $[1,2,7]$ have assessed the amounts of carbon and sulfur brought to the mantle in subduction zones at an annual input of $\sim 4.6 \pm 4.0 \times 10^{12}$ and $\sim 2.5 \pm 1.5 \times 10^{12}$ moles, respectively. Given that the removal of $C$ and $S$ by volcanic arcs is less than the above values, it may be supposed that some amounts of carbon and sulfur stay deep in the mantle where they can interact with mantle minerals. Currently, it is known that carbonate crustal material can be subducted to a depth of $\geq 600 \mathrm{~km}$ [8], since carbonates are thermodynamically stable even at lower mantle P-T parameters [9]. The presence of carbonates in mantle rocks is confirmed by the findings of carbonate inclusions in 
diamonds [10-14]. However, it should be noted that subduction is not the only possible source of sulfur, it can occur as fluids of mantle plume or processes at the core-mantle boundary.

Data on the composition of inclusions in mantle xenolith minerals as well as experimental results indicate that sulfur in the upper mantle, depending on pressure, temperature, and oxygen fugacity, can occur in the form of sulfides or sulfide melts $[8,15,16]$, sulfates $[17,18]$, and C-O-H-S fluid [18-21] or be present in a dissolved form in silicate melts [22-24]. In recent years, the behavior of sulfur in mantle metasomatic processes and the relationship of these processes with formation of sulfide minerals in eclogites and peridotites of the upper mantle have been actively studied [25-27]. In particular, some researchers suggest that sulfides may be formed during the interaction between S-bearing fluids or melts and mantle Fe,Ni-bearing silicates via sulfidation reactions $[15,27-30]$. Olivine sulfidation reactions were described in many settings, such as Kaapvaal craton peridotites [15], silicate rocks of the Merensky Reef (South Africa) [31], and chondrites (Lodran primitive achondrite [29]; enstatite chondrites $[28,30])$. However, Fe-rich carbonate sulfidation was never described in the natural samples. Findings of carbonate-sulfide assemblages in xenoliths were described by [26], and also as inclusions in diamonds [32].

To date, experimental studies simulating sulfidation of olivine, pyroxene, and garnet at crustal pressures have been performed [33,34]. High-pressure experiments on simulation of sulfidation processes in the silicate mantle with involvement of S-bearing fluids or melts and on identification of a potential mechanism of sulfide formation under deep subduction conditions have been performed in olivine-sulfur and olivine-pyrite [35], olivine-Fe,Ni-sulfide [36], and olivine-sulfate-carbon [37] systems. Experiments simulating the behavior of carbon- and sulfur-bearing phases in subduction processes have become topical in recent years; however, these are single studies on the interaction of carbonates and sulfur (a simplified model of subducted marine sediments) with silicate phases at mantle pressures and temperatures. Previously, we experimentally studied the interactions of alkaline earth carbonate, reduced sulfur fluid, and olivine [38]. The main goal of this study is to experimentally simulate the interaction processes between mantle silicates and subducted material, which include sulfidation of Fe-bearing carbonate (ankerite) and Fe,Ni-olivine during the interaction with sulfur melts under mantle P,T-parameters.

\section{Materials and Methods}

Experimental modeling of the interaction between mantle silicates and subducted material was performed in the olivine-ankerite-sulfur $\left((\mathrm{Mg}, \mathrm{Fe}, \mathrm{Ni}) \mathrm{SiO}_{4}-\mathrm{Ca}(\mathrm{Fe}, \mathrm{Mg}, \mathrm{Mn})\left(\mathrm{CO}_{3}\right)_{2}-\mathrm{S}\right.$ and olivine-ankerite-pyrite $\left((\mathrm{Mg}, \mathrm{Fe}, \mathrm{Ni}) \mathrm{SiO}_{4}-\mathrm{Ca}(\mathrm{Fe}, \mathrm{Mg}, \mathrm{Mn})\left(\mathrm{CO}_{3}\right)_{2}-\mathrm{FeS}_{2}\right)$ systems using a multi-anvil high-pressure "split-sphere" apparatus (BARS) [39] at a pressure of $6.3 \mathrm{GPa}$, in a temperature range of $1050-1550^{\circ} \mathrm{C}$, and at the run duration from 20 to $60 \mathrm{~h}$. The methodological features of the assembly and high-pressure cell design as well as the calibration data were published elsewhere [40-42]. Starting materials included natural $\mathrm{Fe}$,Ni-bearing olivine $\left(\mathrm{Mg}_{1.8} \mathrm{Fe}_{0.19} \mathrm{Ni}_{0.01} \mathrm{SiO}_{4}\right.$, granular spinel lherzolite xenolith, Udachnaya pipe, Yakutia), ankerite $\left(\mathrm{CaFe}_{0.49} \mathrm{Mg}_{0.49} \mathrm{Mn}_{0.02}\left(\mathrm{CO}_{3}\right)_{2}\right.$, Kyshtym deposit, Urals), pyrite $\left(\mathrm{FeS}_{2}\right.$, Astaf'evskoe deposit, Urals), and pure sulfur (Table 1). The starting materials were used in the following weight proportions: olivine/ankerite/sulfur $=30 / 30 / 8.5 \mathrm{mg}$ in the olivine-ankerite-sulfur system and olivine/ankerite/pyrite $=33.2 / 33 / 10.5 \mathrm{mg}$ in the olivine-ankerite-pyrite system. This proportion of $\mathrm{Fe}, \mathrm{Ni}$-bearing olivine and sulfur/pyrite were chosen according to the schematic reaction of total extraction of Fe and Ni from olivine to form pyrrhotite. Both pyrite and sulfur were used to compare the results of olivine and carbonate interactions with sulfur melt and with sulfide or sulfide melt.

Given the previous experience in studying sulfides and sulfur at mantle P and T [21,35], we chose graphite as the optimal material for capsules. A scheme of the graphite capsule is shown in Figure 1a. We used an ampoule assembly design where starting materials were finely crushed and homogenized. Three diamond seed crystals with a cubo-octahedral habit ( $500 \mu \mathrm{m}$ in size) were placed in the sample to investigate diamond growth under the experimental parameters. 
Table 1. Compositions of starting materials (wt. \%).

\begin{tabular}{lcccccccccc}
\hline Mineral & Si & Ca & Fe & Mg & Mn & Ni & S & C & O & Total \\
\hline Olivine & 19.17 & - & 7.22 & 29.43 & - & 0.35 & - & - & 43.96 & 99.86 \\
Ankerite & - & 19.27 & 13.7 & 5.87 & 0.55 & - & - & 11.98 & 47.93 & 100 \\
Pyrite & - & - & 46.82 & - & - & 0.03 & 53.04 & - & - & 99.89 \\
\hline
\end{tabular}

Samples produced in the experiments were impregnated with epoxy, sawn (Figure 1b), and polished. The primary study of samples was performed using Stemi 508 and Axio Imager 2 stereomicroscopes (Carl Zeiss Microscopy, Jena, Germany). Phase and chemical compositions of samples as well as phase relationships were studied by microprobe analysis (Camebax-Micro, Cameca, Gennevilliers, France) and electron scanning microscopy (TESCAN MIRA 3 LMU, Tescan, Brno, Czech Republic). An analysis of silicate phases was carried out at an accelerating voltage of $20 \mathrm{kV}$, a probe current of $20 \mathrm{nA}$, a counting time of $10 \mathrm{~s}$ in each analytical line, and a probe electron beam diameter of 2 to $3 \mu \mathrm{m}$.
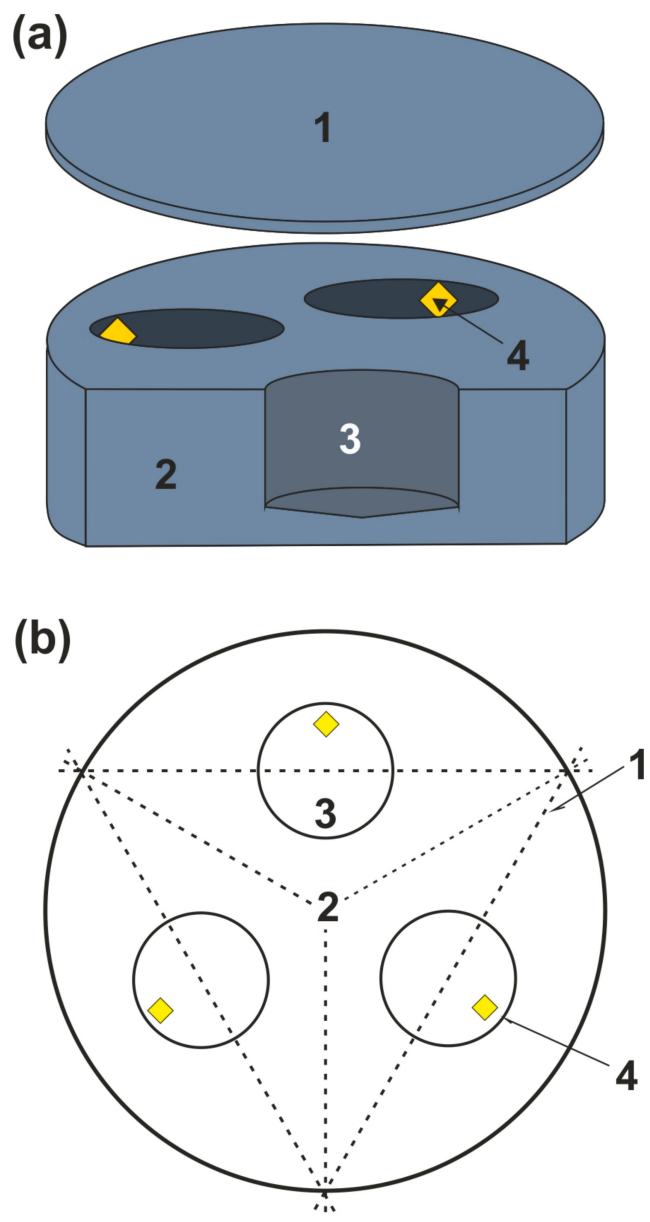

Figure 1. Scheme of a graphite capsule (a): 1-graphite cover; 2-graphite container with three blind holes; 3-sample volume; 4-diamond seed crystals; (b) - Scheme for sawing of samples (top view): 1-sawing lines, 2-graphite container, 3-sample, 4-diamond seed crystals.

The electron beam diameter was increased to $20 \mu \mathrm{m}$ to analyze the compositions of quenched melts that were represented by microdendritic aggregates. The morphology of diamond seeds was studied by differential interference contrast microscopy and scanning electron microscopy. Analytical studies were performed at the Center for Collective Use of Multi-element and Isotopic Analysis of the Siberian Branch of Russian Academy of Sciences. In addition, Raman spectroscopy (Jobin Yvon 
LabRAM HR800 spectrometer, Horiba, Japan, equipped with an Olympus BX41 stereo microscope, Olympus, Tokyo, Japan) was used to study structural features of silicates, carbonates, and sulfides as well as the composition of inclusions in olivine crystals. A He-Cd laser at a wavelength of $325 \mathrm{~nm}$ was used as an excitation source.

\section{Results}

\subsection{Olivine-Ankerite-Sulfur System}

The experimental results are presented in Table 2. Experimental study of the olivine-ankerite-sulfur interactions demonstrated that samples in relatively low-temperature experiments $\left(\mathrm{T}=1050-1250{ }^{\circ} \mathrm{C}\right.$, $\mathrm{t}=60 \mathrm{~h}$ ) were represented by an aggregate of coarse-crystalline olivine $(0.1-1.0 \mathrm{~mm}$ ) and pyrite (up to $100 \mu \mathrm{m}$ ), the interstices of which contained fine-crystalline olivine, diopside, magnesite, and aragonite (Figures $2 \mathrm{a}, \mathrm{b}$ and $3 \mathrm{a}$ ) as well as a microdendritic aggregate of sulfur. According to the published data $[35,43,44]$, at $6.3 \mathrm{GPa}$ and temperatures of $1050-1250^{\circ} \mathrm{C}$, sulfur occurs in a molten state, and pyrite is stable (Figure 4). Given this fact, the microdendritic sulfur aggregate may be considered as quenched sulfur melt. In a range of $1050-1250{ }^{\circ} \mathrm{C}$, samples contain zonal aggregates (Figures $2 \mathrm{~b}-\mathrm{d}$ and $3 \mathrm{a}^{*}$ ) consisting of relatively high-iron olivine with rims of Fe-poor olivine with clinopyroxene and pyrite inclusions, which are surrounded by $\mathrm{Ms}+\mathrm{Py} \rightarrow \mathrm{Cpx}+\mathrm{Arg}+\mathrm{Py}$ reaction zones. The produced olivine crystals exhibit signs of recrystallization in a sulfur melt, the main evidence of which is: (i) an increased dimension of olivine from $\sim 20 \mu \mathrm{m}$ (at the start) to $\sim 1 \mathrm{~mm}$; (ii) a zonal structure of crystals, with an iron-rich core $\left(\mathrm{Mg}_{1.81} \mathrm{Fe}_{0.18} \mathrm{Ni}_{0.01} \mathrm{SiO}_{4}\right)$ and a magnesium-rich rim $\left(\mathrm{Mg}_{1.99} \mathrm{Fe}_{0.01} \mathrm{SiO}_{4}\right)$ (Table 3); (iii) formation of pyrite and clinopyroxene inclusions in olivine (Figure 2c-e). The composition profiles of olivine produced at $1050{ }^{\circ} \mathrm{C}$ are shown in Figure $5 \mathrm{a}, \mathrm{b}$. Between the rims and the cores of olivine, there are characteristic sharp boundaries with a drop in the concentrations of $\mathrm{FeO}$ and $\mathrm{NiO}$ from $\sim 9 \mathrm{wt} . \%$ and 0.45 wt. \%, respectively, to zero. The concentration of $\mathrm{FeO}$ in newly formed diopside $\left(\mathrm{Mg}_{1.3} \mathrm{Ca}_{0.7} \mathrm{Si}_{2} \mathrm{O}_{6}\right)$ (Table 3) and carbonates, which are represented by magnesite, dolomite, and aragonite, does not exceed $1 \mathrm{wt}$. \% (Table 4). Newly formed pyrite contains $\sim 1.5 \mathrm{wt}$. \% of Ni (Table 5 ). The sulfur melt contains $\sim 10$ wt. \% of dissolved components: $\mathrm{Fe}, \mathrm{Ni}$, and $\mathrm{O}$ (Table 5).

Table 2. Experimental parameters and results in the olivine-ankerite-sulfur and olivine-ankerite-pyrite systems at $6.3 \mathrm{GPa}$.

\begin{tabular}{|c|c|c|c|c|c|c|}
\hline \multirow{2}{*}{ Run N } & \multirow{2}{*}{$\mathrm{T}^{\circ} \mathrm{C}$} & \multirow{2}{*}{$t, h$} & \multicolumn{4}{|c|}{ Final Phases } \\
\hline & & & Silicates & Carbonates & S-Bearing Phases & Phases of Elemental Carbon \\
\hline \multicolumn{7}{|c|}{ Olivine-Ankerite-Sulfur System } \\
\hline $932-3$ & 1050 & 60 & $\mathrm{Ol}, \mathrm{Cpx}$ & Dol, Mgs, Arg & Py, Ls & - \\
\hline $1476-3$ & 1150 & 60 & $\mathrm{Ol}, \mathrm{Cpx}$ & Dol, Mgs, Arg & Py, Ls & - \\
\hline $1997-3$ & 1250 & 60 & $\mathrm{Ol}, \mathrm{Cpx}$ & Dol, Mgs & Py, Ls & - \\
\hline $2000-3$ & 1350 & 20 & $\mathrm{Ol}, \mathrm{Cpx}$ & Dol, Mgs & Ls & Gr \\
\hline $2001-3$ & 1450 & 20 & $\mathrm{Ol}, \mathrm{Opx}$ & Mgs, Dol, $\mathrm{L}_{\text {carb-sil }}$ & Ls & Gr \\
\hline $1181-3$ & 1550 & 20 & $\mathrm{Ol}$ & $\mathrm{L}_{\text {carb-sil }}$ & Ls & Gr, Dm \\
\hline \multicolumn{7}{|c|}{ Olivine-Ankerite-Pyrite System } \\
\hline $932-6$ & 1050 & 60 & $\mathrm{Ol}, \mathrm{Cpx}$ & Dol, Mgs & Py, Po & - \\
\hline $1476-6$ & 1150 & 60 & $\mathrm{Ol}, \mathrm{Cpx}$ & Dol, Mgs & Py, Po & - \\
\hline $1997-6$ & 1250 & 60 & $\mathrm{Ol}, \mathrm{Cpx}$ & Dol, Mgs & Py, Po & - \\
\hline $2000-6$ & 1350 & 20 & $\mathrm{Ol}, \mathrm{Cpx}$ & Dol, Mgs, $\mathrm{L}_{\mathrm{carb}}$ & $\mathrm{L}_{\mathrm{Fe}-\mathrm{S}-\mathrm{O}}$ & - \\
\hline $2001-6$ & 1450 & 20 & $\mathrm{Ol}, \mathrm{Opx}$ & Mgs, $\mathrm{L}_{\text {carb }}$ & $\mathrm{L}_{\mathrm{Fe}-\mathrm{S}-\mathrm{O}}$ & Gr \\
\hline $1181-6$ & 1550 & 20 & $\mathrm{Ol}$ & $\mathrm{L}_{\text {carb }}$ & $\mathrm{L}_{\mathrm{Fe}-\mathrm{S}-\mathrm{O}}$ & Gr, Dm \\
\hline
\end{tabular}

Ol-olivine, Cpx-clinopyroxene, Opx-orthopyroxene, Dol-dolomite, Mgs-magnesite, Arg-aragonite, Py-pyrite, Po-pyrrhotite, Ls-sulfur melt with dissolved components, $\mathrm{L}_{\text {carb-sil }}$ carbonate-silicate melt,

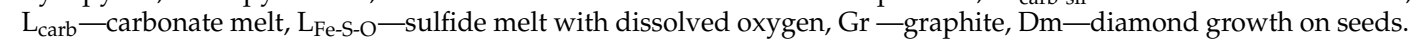



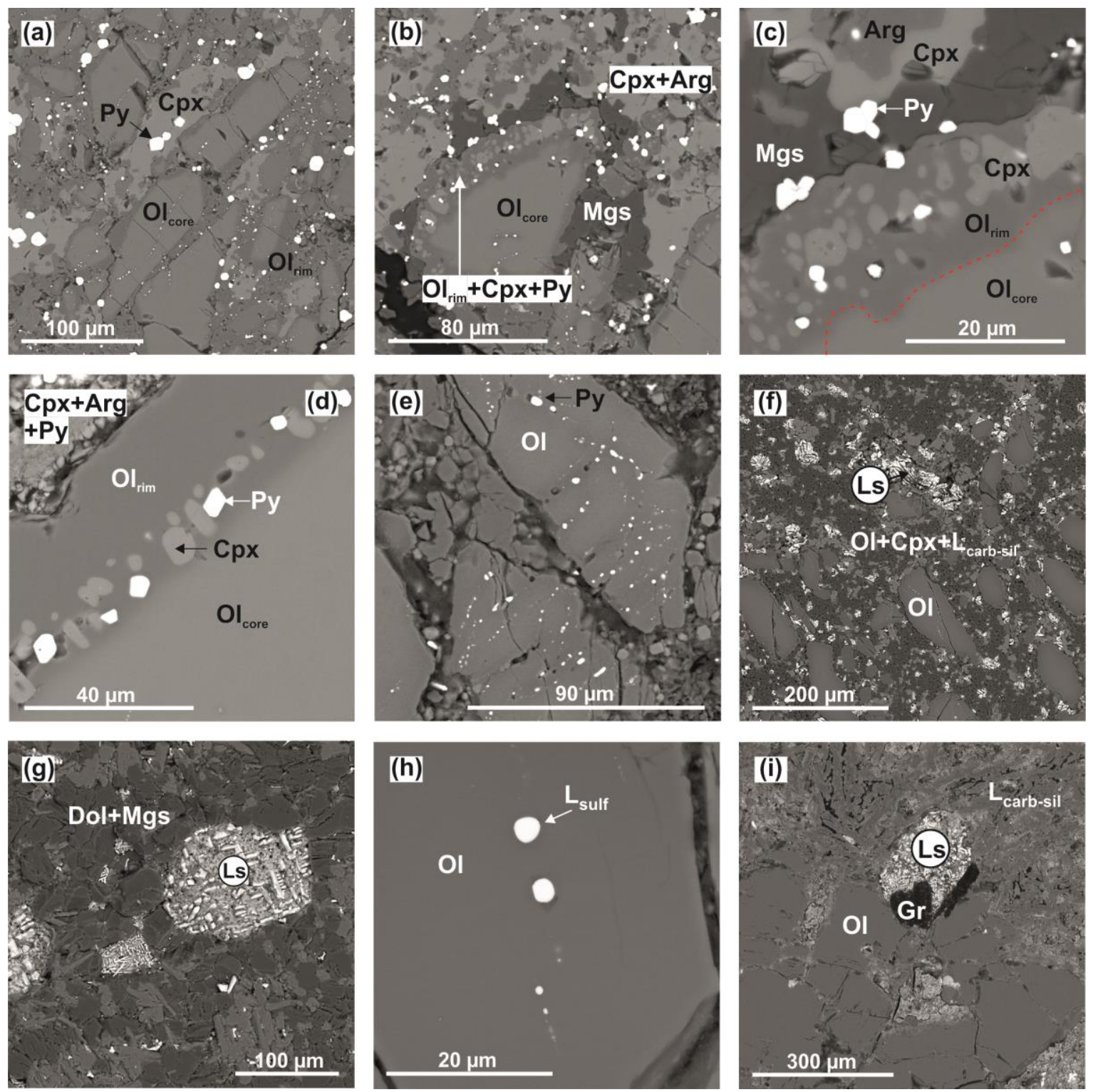

Figure 2. Scanning electron microscope (SEM) micrographs of polished fragments of samples produced in the olivine-ankerite-sulfur system: $(\mathbf{a}, \mathbf{b})$ - polycrystalline olivine aggregate with fine-crystalline olivine, clinopyroxene, magnesite, and aragonite in the interstices $\left(1050^{\circ} \mathrm{C}\right)$; (c) - structure of the reaction zones around recrystallized olivine $\left(1050^{\circ} \mathrm{C}\right),(\mathrm{d})$ - pyrite and diopside inclusions in recrystallized olivine $\left(1150{ }^{\circ} \mathrm{C}\right)$; (e) —recrystallized olivine with pyrite inclusions $\left(1250{ }^{\circ} \mathrm{C}\right)$; $(\mathbf{f})$ - polycrystalline aggregate of recrystallized olivine, clinopyroxene, and dolomite, as well as pools of a quenched sulfur melt $\left(1350{ }^{\circ} \mathrm{C}\right) ;(\mathrm{g})$-droplets of a quenched sulfur melt in a polycrystalline dolomite + magnesite aggregate $\left(1450{ }^{\circ} \mathrm{C}\right)$; (h) —recrystallized olivine with sulfide inclusions $\left(1450{ }^{\circ} \mathrm{C}\right)$; (i) —large crystals of graphite at the contact between recrystallized olivine and quenched sulfur- and carbonate-silicate melts $\left(1550{ }^{\circ} \mathrm{C}\right)$; Ol-olivine, Cpx-clinopyroxene, Py-pyrite, Mgs-magnesite, Arg-aragonite,

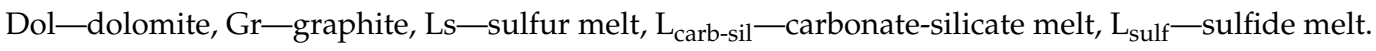




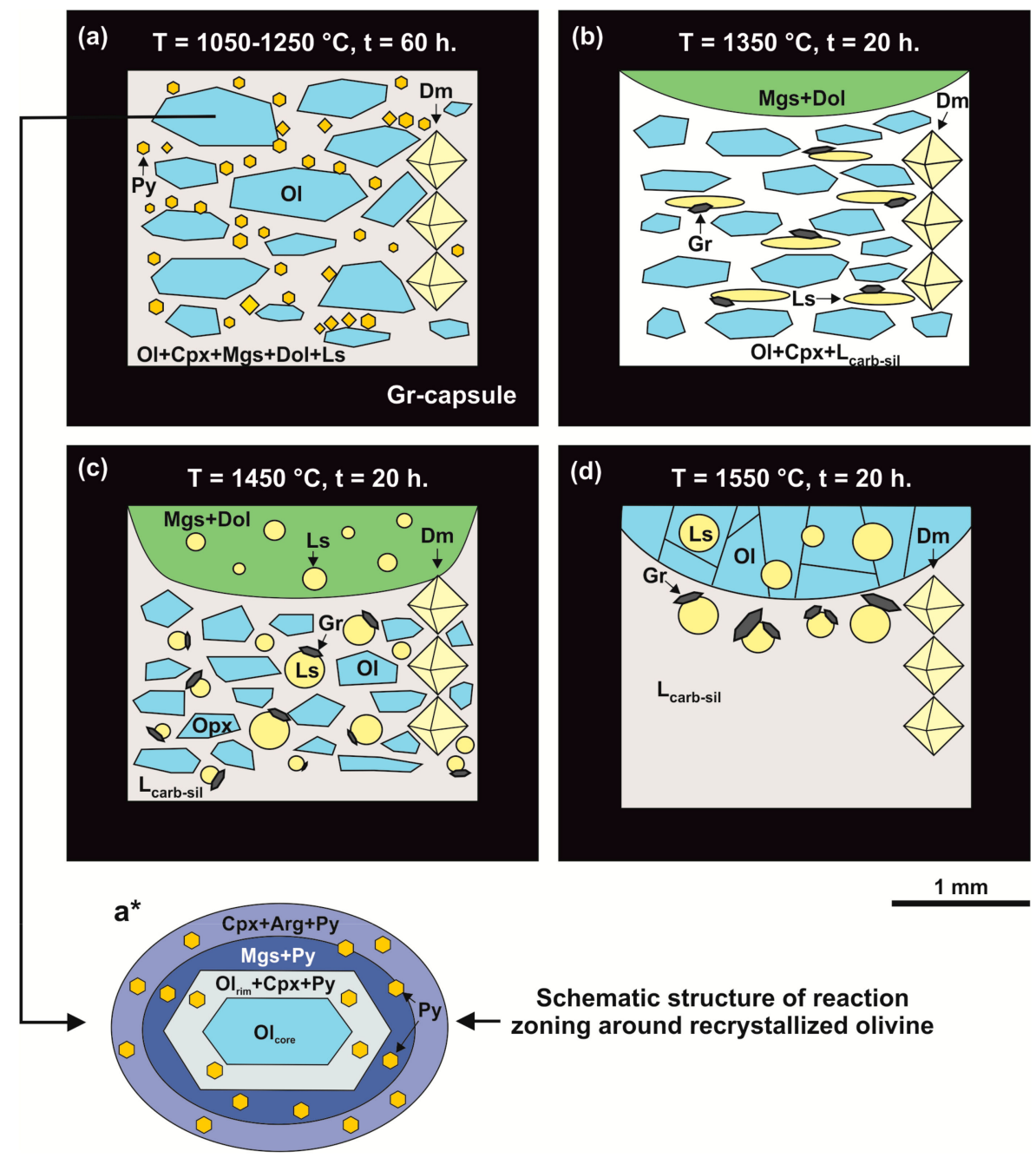

Figure 3. Schemes of samples produced in the olivine-ankerite-sulfur system at pressure of $6.3 \mathrm{GPa}$ in a temperature range of $1050-1550{ }^{\circ} \mathrm{C} .(\mathbf{a}): \mathrm{T}=1050-1250{ }^{\circ} \mathrm{C} ;(\mathbf{b}): \mathrm{T}=1350{ }^{\circ} \mathrm{C} ;(\mathbf{c}): \mathrm{T}=1450{ }^{\circ} \mathrm{C}$; (d): $\mathrm{T}=1550{ }^{\circ} \mathrm{C} ;\left(\mathbf{a}^{*}\right)$ : schematic structure of reaction zoning around recrystallized olivine. Py-pyrite, $\mathrm{Ol}$-olivine, Opx—orthopyroxene, Mgs—magnesite, Dol—dolomite, Gr—graphite, Ls—sulfur melt,

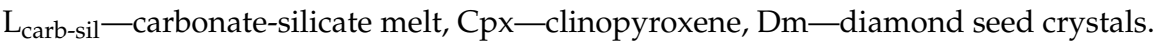

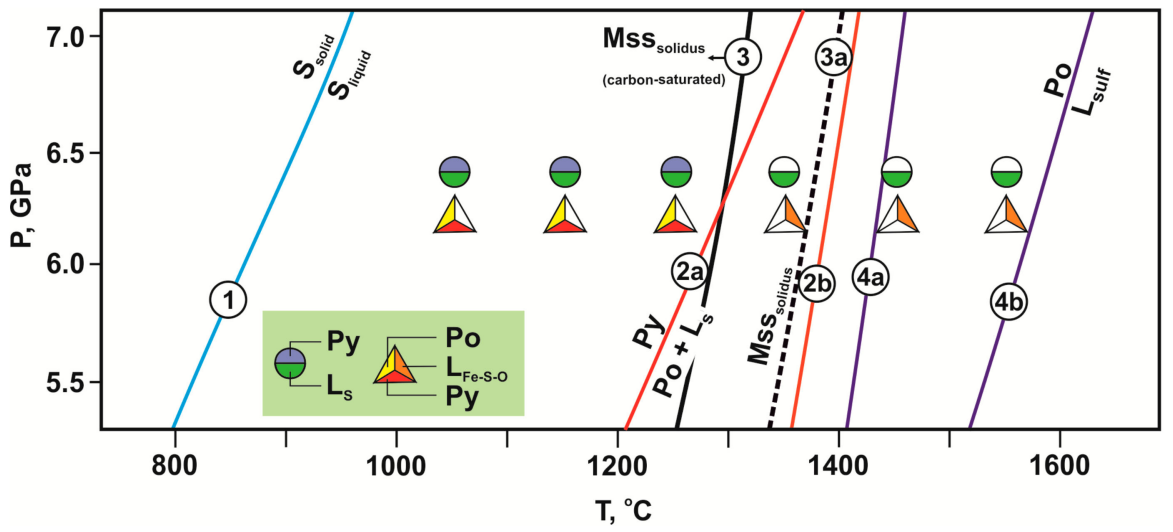

Figure 4. P-T diagram with parameters and results of experiments in the olivine-ankerite-sulfur (circles) and olivine-ankerite-pyrite (triangles) systems. Symbols are vertically displaced for clarity. Experimentally determined curves: 1-melting of sulfur [43]; 2a, 4a-decomposition of pyrite [44,45]; 2b, 4b-melting of pyrrhotite [44,45]; 3, 3a—solidi of monosulfide solid solution [46,47]. 
Table 3. Averaged compositions of silicates.

\begin{tabular}{|c|c|c|c|c|c|c|c|c|c|c|c|c|c|c|c|}
\hline \multirow{2}{*}{ Run N } & \multirow{2}{*}{$\mathrm{T},{ }^{\circ} \mathrm{C}$} & \multirow{2}{*}{ Phase } & \multicolumn{6}{|c|}{ Mass Concentrations. wt. \% } & \multirow{2}{*}{$\mathrm{n}(\mathrm{O})$} & \multicolumn{6}{|c|}{ Cations Per Formula Unit } \\
\hline & & & $\mathrm{SiO}_{2}$ & $\mathrm{FeO}$ & $\mathrm{MgO}$ & $\mathrm{CaO}$ & $\mathrm{NiO}$ & Total & & $\mathrm{Si}$ & $\mathrm{Fe}$ & $\mathrm{Mg}$ & $\mathrm{Ca}$ & $\mathrm{Ni}$ & Sum \\
\hline \multicolumn{16}{|c|}{ Olivine-Ankerite-Sulfur System } \\
\hline \multirow{4}{*}{$932-3$} & & $\mathrm{Ol}_{\text {core }}$ & $40.5_{(3)}$ & $9.1_{(3)}$ & $49.2_{(2)}$ & $0.07_{(7)}$ & $0.45_{(2)}$ & 99.47 & 4 & $1.00_{(0)}$ & $0.19_{(1)}$ & $1.81_{(1)}$ & - & $0.01_{(0)}$ & 3.01 \\
\hline & 1050 & $\mathrm{Ol}_{\text {rim }}$ & $42.3(2)$ & $0.1_{(1)}$ & $56.8_{(3)}$ & $0.3_{(2)}$ & - & 99.62 & 4 & $0.99(0)$ & $-(1)$ & $2.00_{(1)}$ & $0.01_{(1)}$ & - & 3.01 \\
\hline & & Cpx & $54.6_{(9)}$ & $0.5_{(5)}$ & $22(3)$ & $22(3)$ & - & 99.57 & 6 & $1.96_{(4)}$ & $0.01_{(1)}$ & $1.2_{(2)}$ & $0.9_{(1)}$ & - & 4.04 \\
\hline & & $\mathrm{Ol}_{\text {core }}$ & $40.6_{(3)}$ & $9.0_{(7)}$ & $49.3_{(6)}$ & $0.02_{(2)}$ & $0.45_{(2)}$ & 99.46 & 4 & $1.00_{(0)}$ & $0.19_{(2)}$ & $1.81_{(1)}$ & - & $0.01_{(0)}$ & 3.01 \\
\hline \multirow[t]{3}{*}{$1476-3$} & 1150 & $\mathrm{Ol}_{\text {rim }}$ & $42.3_{(3)}$ & $0.6_{(6)}$ & $56.6_{(7)}$ & $0.06_{(6)}$ & - & 99.62 & 4 & $1.00_{(1)}$ & $0.01_{(1)}$ & $2.00_{(2)}$ & - & - & 3.00 \\
\hline & & Cpx & $54.3(6)$ & $0.5(1)$ & $22_{(3)}$ & $22_{(3)}$ & - & 99.76 & 6 & $1.95_{(3)}$ & $0.01_{(0)}$ & $1.2(1)$ & $0.9_{(1)}$ & - & 4.03 \\
\hline & & $\mathrm{Ol}_{\text {core }}$ & $40.8_{(4)}$ & $8.7_{(9)}^{17}$ & $49.5_{(9)}$ & $0.06_{(6)}$ & $0.45_{(3)}$ & 99.52 & 4 & $1.00_{(0)}$ & $0.18_{(2)}$ & $1.82_{(2)}$ & - & $0.01_{(0)}$ & 3.01 \\
\hline \multirow[t]{3}{*}{$1997-3$} & 1250 & $\mathrm{Ol}_{\text {rim }}$ & $41.3_{(5)}$ & $2.9_{(1)}$ & $54.4_{(3)}$ & $0.1_{(1)}$ & $0.3_{(0)}$ & 99.16 & 4 & $0.99_{(1)}$ & $0.06_{(0)}$ & $1.95_{(2)}$ & - & $0.01_{(0)}$ & 3.01 \\
\hline & & Cpx & $54.9(6)$ & $0.4_{(2)}$ & $20.5(9)$ & $23(1)$ & - & 99.55 & 6 & $1.98_{(1)}$ & $0.01_{(1)}$ & $1.11_{(5)}$ & $0.92_{(5)}$ & - & 4.02 \\
\hline & & $\mathrm{Ol}_{\text {core }}$ & $40.8_{(4)}$ & $8.5_{(9)}$ & $49.7_{(8)}$ & $0.02_{(2)}$ & $0.45_{(2)}$ & 99.49 & 4 & $1.00_{(1)}$ & $0.17_{(2)}$ & $1.83_{(2)}$ & - & $0.01_{(0)}$ & 3.01 \\
\hline \multirow[t]{3}{*}{$2000-3$} & 1350 & $\mathrm{Ol}_{\text {rim }}$ & $41.9_{(1)}$ & $1.7_{(5)}$ & $55.6_{(4)}$ & $0.2_{(1)}$ & $0.1_{(1)}$ & 99.66 & 4 & $0.99_{(0)}$ & $0.03_{(1)}$ & $1.97_{(1)}^{(2)}$ & $0.01_{(0)}$ & - & 3.01 \\
\hline & & Cpx & $55_{(1)}$ & $0.2_{(2)}$ & $21.8_{(7)}$ & $22.6_{(8)}$ & - & 99.75 & 6 & $1.98_{(3)}$ & $0.01_{(0)}$ & $1.18_{(4)}$ & $0.87_{(4)}$ & - & 4.02 \\
\hline & & $\mathrm{Ol}_{\text {core }}$ & $40.8_{(4)}$ & $6_{(1)}$ & $52_{(1)}$ & $0.02_{(2)}$ & $0.4_{(1)}$ & 99.67 & 4 & $0.99_{(0)}$ & $0.13_{(3)}$ & $1.88_{(4)}$ & - & $0.01_{(0)}$ & 3.01 \\
\hline \multirow{2}{*}{ 2001-3 } & 1450 & $\mathrm{Ol}_{\text {rim }}$ & $41.9_{(5)}$ & $1.5_{(9)}$ & $56.1_{(9)}$ & $0.1_{(1)}$ & $0.1_{(1)}$ & 99.64 & 4 & $0.99_{(1)}$ & $0.03_{(2)}$ & $1.99_{(2)}$ & - & - & 3.01 \\
\hline & & Opx & $58.8(5)$ & $0.3_{(1)}$ & $38.1_{(6)}$ & $2.4_{(4)}$ & - & 99.62 & 6 & $1.99(1)$ & $0.01_{(0)}$ & $1.93_{(3)}$ & $0.09_{(1)}$ & - & 4.01 \\
\hline $1811-3$ & 1550 & Ol & $42.5_{(3)}$ & $0.3_{(2)}$ & $56.5_{(2)}$ & $0.1_{(1)}$ & - & 99.37 & 4 & $1.00_{(0)}$ & $0.01_{(0)}$ & $1.99(1)$ & - & - & 3.00 \\
\hline \multicolumn{16}{|c|}{ Olivine-Ankerite-Pyrite System } \\
\hline \multirow{4}{*}{$932-6$} & & $\mathrm{Ol}_{\text {core }}$ & $40.6_{(2)}$ & $9.2_{(2)}$ & $49.1_{(1)}$ & - & $0.44_{(8)}$ & 99.5 & 4 & $1.00_{(0)}$ & $0.19_{(0)}$ & $1.81_{(1)}$ & - & $0.01_{(0)}$ & 3.00 \\
\hline & 1050 & $\mathrm{Ol}_{\text {rim }}$ & $43_{(1)}$ & $1.4_{(5)}$ & $54_{(2)}$ & - & - & 99.8 & 4 & $1.01_{(2)}$ & $0.03_{(1)}$ & $1.91_{(9)}$ & - & - & 2.99 \\
\hline & & Cpx & $55.4_{(6)}$ & $0.6_{(4)}$ & $19.2(5)$ & $24.4_{(3)}$ & - & 99.8 & 6 & $2.00_{(2)}$ & $0.02_{(1)}$ & $1.04_{(3)}$ & $0.94_{(1)}$ & - & 4.00 \\
\hline & & $\mathrm{Ol}_{\text {core }}$ & $40.5_{(4)}$ & $9.3_{(6)}$ & $49.3_{(4)}$ & - & $0.49_{(6)}$ & 99.6 & 4 & $0.99_{(1)}$ & $0.19_{(1)}$ & $1.81_{(1)}$ & - & $0.01_{(0)}$ & 3.01 \\
\hline \multirow[t]{3}{*}{$1476-6$} & 1150 & $\mathrm{Ol}_{\text {rim }}$ & $41.4_{(6)}$ & $3_{(1)}$ & $53_{(1)}$ & - & - & 99.3 & 4 & $0.99_{(1)}$ & $0.07_{(2)}$ & $1.91_{(5)}$ & - & - & 3.01 \\
\hline & & Cpx & $55.0_{(2)}$ & $0.8_{(3)}$ & $18.8_{(3)}$ & $24.7_{(3)}$ & - & 100,0 & 6 & $2.00_{(1)}$ & $0.02_{(1)}$ & $1.02_{(2)}$ & $0.96_{(1)}$ & - & 4.00 \\
\hline & & $\mathrm{Ol}_{\text {core }}$ & $41.0_{(3)}$ & $9(1)$ & $50_{(1)}$ & - & $0.42_{(8)}$ & 99.5 & 4 & $1.00_{(0)}$ & $0.18_{(3)}$ & $1.81_{(3)}$ & - & $0.01_{(0)}$ & 3.00 \\
\hline \multirow[t]{3}{*}{$1997-6$} & 1250 & $\mathrm{Ol}_{\text {rim }}$ & $42.2(5)$ & $3_{(1)}$ & $54.3_{(9)}$ & - & - & 99.7 & 4 & $1.00_{(1)}$ & $0.06_{(2)}$ & $1.94_{(2)}$ & - & - & 3.00 \\
\hline & & Cpx & $55_{(1)}$ & $1.0_{(6)}$ & $21_{(1)}$ & $23_{(1)}$ & - & 99.5 & 6 & $1.98_{(4)}$ & $0,03_{(2)}$ & $1.12_{(7)}$ & $0.89_{(6)}$ & - & 4,02 \\
\hline & & $\mathrm{Ol}_{\text {core }}$ & $41.0_{(3)}$ & $8_{(1)}$ & $50_{(1)}$ & - & $0.47(9)$ & 99.7 & 4 & $1.00_{(0)}$ & $0.16_{(2)}$ & $1.83_{(2)}$ & - & $0.01_{(0)}$ & 2.99 \\
\hline \multirow[t]{3}{*}{$2000-6$} & 1350 & $\mathrm{Ol}_{\text {rim }}$ & $42.0_{(5)}$ & $3_{(1)}$ & $54_{(1)}$ & - & - & 99,7 & 4 & $1.00_{(1)}$ & $0.06_{(3)}$ & $1.93_{(3)}$ & - & - & 3.00 \\
\hline & & Cpx & $55.4_{(6)}$ & $0.9_{(5)}$ & $21.3_{(7)}$ & $22.2_{(9)}$ & - & 99.8 & 6 & $1.99_{(1)}$ & $0.03_{(1)}$ & $1.14_{(4)}$ & $0.85_{(4)}$ & - & 4.01 \\
\hline & & $\mathrm{Ol}_{\text {core }}$ & $40.9_{(5)}^{(b)}$ & $8_{(2)}$ & $51_{(1)}$ & - & $0,5_{(1)}$ & 99.9 & 4 & $1.00_{(1)}$ & $0.16_{(3)}$ & $1.85_{(3)}$ & - & $0.01_{(0)}$ & 3.00 \\
\hline \multirow[t]{2}{*}{$2001-6$} & 1450 & $\mathrm{Ol}_{\text {rim }}$ & $41.8_{(4)}$ & $3.8_{(6)}$ & $53.9_{(6)}$ & - & - & 99.8 & 4 & $1.00_{(1)}$ & $0.08_{(1)}$ & $1.92_{(2)}$ & - & - & 3.00 \\
\hline & & Opx & $58.3_{(7)}$ & $2.1_{(2)}$ & $36.7_{(9)}$ & $2.9_{(9)}$ & - & 100.1 & 6 & $1.99_{(1)}$ & $0.06_{(1)}$ & $1.86_{(4)}$ & $0.10_{(3)}$ & - & 4.01 \\
\hline \multirow{2}{*}{$1811-6$} & & $\mathrm{Ol}_{\text {core }}$ & $41.3_{(3)}$ & $6_{(1)}$ & $52_{(1)}$ & - & - & 99.6 & 4 & $1.00_{(1)}$ & $0.12_{(2)}$ & $1.88_{(3)}$ & - & - & 3.00 \\
\hline & 1550 & $\mathrm{Ol}_{\text {rim }}$ & $42.4_{(2)}$ & $0.41_{(8)}$ & $56.5_{(5)}$ & - & - & 99,6 & 4 & $1.00_{(0)}$ & $0.01_{(0)}$ & $1.99_{(2)}$ & - & - & 3.00 \\
\hline
\end{tabular}

$\mathrm{Ol}$-olivine, $\mathrm{Cpx}$ —clinopyroxene, Opx—orthopyroxene; $\mathrm{n}(\mathrm{O})$-number of the oxygen atoms; the values in parentheses are one sigma errors of the means based on replicate electron microprobe analyses reported as least units cited; $4.3_{(1)}$ should be read as $4.3 \pm 0.1 \mathrm{wt}$. \%. 

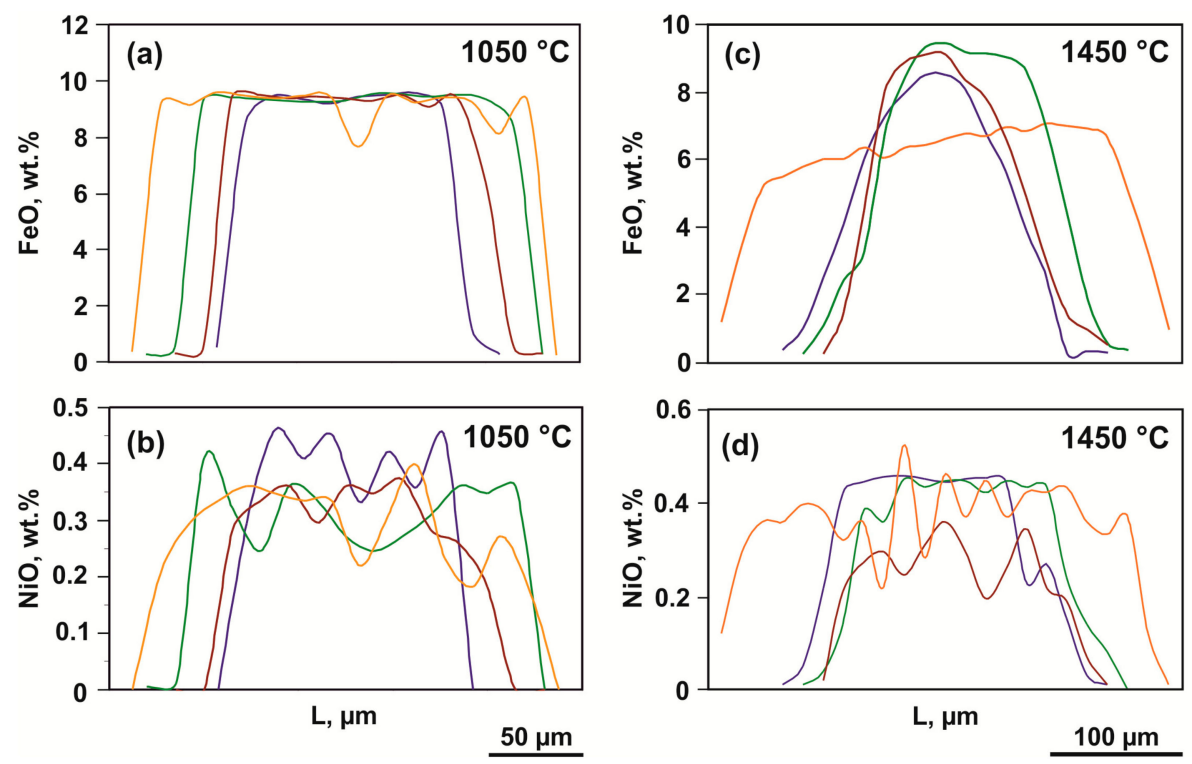

Figure 5. Representative profiles of the $\mathrm{FeO}$ and $\mathrm{NiO}$ distribution in recrystallized olivine (olivine-ankerite-sulfur system): (a,b) $-1050{ }^{\circ} \mathrm{C} ;(\mathbf{c}, \mathbf{d})-1450{ }^{\circ} \mathrm{C}$.

The olivine-ankerite-sulfur interaction at a temperature of $1350{ }^{\circ} \mathrm{C}(\mathrm{t}=20 \mathrm{~h})$ was found to result in the formation of a coarse-crystalline aggregate of recrystallized olivine (Figure 2), with a fine-crystalline matrix of olivine and diopside in interstices as well as asymmetric droplets of a quenched sulfur melt. Single graphite crystals are formed at the boundary of sulfur melt droplets with a fine-crystalline carbonate-bearing matrix. The upper part of the samples is represented by magnesite and dolomite (Figure 3b). Pyrite is absent in the produced samples, which agrees well with the data $[35,44]$ that pyrite undergoes incongruent melting at $6.3 \mathrm{GPa}$ and $\mathrm{T}>1250{ }^{\circ} \mathrm{C}$ (Figure 4$)$. At $1450{ }^{\circ} \mathrm{C}(\mathrm{t}=20 \mathrm{~h}$ ), the sample is presented by a quenched carbonate-silicate melt, comprising recrystallized olivine $(\sim 0.5 \mathrm{~mm}$ in size), newly formed orthopyroxene and graphite, and droplets of a sulfur melt (Figures $2 \mathrm{~g}$ and $3 \mathrm{c}$ ). At $15500^{\circ} \mathrm{C}(\mathrm{t}=20 \mathrm{~h})$, the upper part of the sample is occupied by an aggregate of recrystallized olivine containing sulfide inclusions (Figure $2 \mathrm{~h}$ ) and relatively large droplets of a sulfur melt (Figure $3 \mathrm{~d}$ ). The lower part of the sample is represented by a quenched carbonate-silicate melt with segregated sulfur melt droplets. Graphite crystals are present on the boundary of two melts (Figure 2i). It should be noted that the olivine-ankerite-sulfur interaction at $1550{ }^{\circ} \mathrm{C}$ leads, apart from graphite formation, to the initial stage of diamond growth on diamond seed crystals.

In a range of $1350-1550{ }^{\circ} \mathrm{C}$, the compositions of produced phases consistently change depending on the temperature. At 1350 and $1450{ }^{\circ} \mathrm{C}$, recrystallized olivine is characterized by a zonal structure, with the FeO concentration in the core of crystals amounting to $6-8 \mathrm{wt}$. $\%$, and the rims being almost iron-free and nickel-free (Table 3). The $\mathrm{FeO}$ and $\mathrm{NiO}$ distribution profiles in zonal crystals produced at $1450{ }^{\circ} \mathrm{C}$ are presented in Figure $5 \mathrm{c}$, d; they differ significantly from the relatively low-temperature ones. A decrease in the iron content from the cores to the rims occurs gradually, which excludes identification of a clear boundary between the zones. In this case, the size of iron-rich cores is much less than the width of low-iron rims. At $1550{ }^{\circ} \mathrm{C}$, recrystallized olivine does not exhibit a zonal structure, and its crystals are homogeneous and compositionally correspond to nickel-free forsterite with $\mathrm{FeO}$ and $\mathrm{CaO}$ impurity contents of 0.3 and $0.1 \mathrm{wt}$ \%, respectively.

Newly formed carbonates (magnesite and dolomite) are characterized by $\mathrm{FeO}$ impurities of $<1 \mathrm{wt}$. \%, with magnesite containing 5-8 wt. \% of $\mathrm{CaO}$. A carbonate-silicate melt contains $10-15 \mathrm{wt}$. \% of $\mathrm{SiO}_{2}$ and low levels of dissolved sulfur and $\mathrm{FeO}$ (up to 1 wt. \%). At $1350-1550{ }^{\circ} \mathrm{C}$, the iron content in a sulfur melt with dissolved components ( $\mathrm{Fe}, \mathrm{Ni}, \mathrm{Si}, \mathrm{Mn}, \mathrm{Mg}, \mathrm{Ca}, \mathrm{C}, \mathrm{O}$ ) is almost constant and amounts to $\sim 22 \mathrm{wt}$. \%. Temperature increase is accompanied by an increase in the contents of carbon (from 3 to $6 \mathrm{wt}$. \%) and oxygen in this melt (from 8 to $16 \mathrm{wt}$. \%) (Table 5). 
Table 4. Averaged compositions of carbonates and carbonate-based melts.

\begin{tabular}{|c|c|c|c|c|c|c|c|c|c|c|c|c|c|c|c|}
\hline \multirow{2}{*}{ Run N } & \multirow{2}{*}{$\mathrm{T},{ }^{\circ} \mathrm{C}$} & \multirow{2}{*}{ Phase } & \multicolumn{7}{|c|}{ Mass Concentrations. wt. $\%$} & \multirow{2}{*}{$\mathrm{n}(\mathrm{O})$} & \multicolumn{5}{|c|}{ Cations Per Formula Unit } \\
\hline & & & $\mathrm{SiO}_{2}$ & $\mathrm{FeO}$ & $\mathrm{MgO}$ & $\mathrm{CaO}$ & $\mathrm{SO}_{3}$ & $\mathrm{CO}_{2} *$ & Total & & $\mathrm{Fe}$ & $\mathrm{Mg}$ & $\mathrm{Ca}$ & $\mathrm{C}$ & Sum \\
\hline \multicolumn{16}{|c|}{ Olivine-Ankerite-Sulfur System } \\
\hline \multirow{3}{*}{$932-3$} & \multirow{3}{*}{1050} & Arg & - & $0.1_{(1)}$ & $0.1_{(1)}$ & $54.2_{(9)}$ & - & $45.7_{(9)}$ & 100 & 3 & - & - & $0.91_{(1)}$ & $1.04_{(1)}$ & 1.96 \\
\hline & & Mgs & - & $0.1_{(1)}$ & $43_{(1)}$ & $3.6_{(1)}$ & - & $52_{(1)}$ & 100 & 3 & - & $0.92_{(3)}$ & $0.05_{(0)}$ & $1.01_{(1)}$ & 1.99 \\
\hline & & Dol & - & - & $19(2)$ & $32_{(3)}$ & - & $47_{(1)}$ & 100 & 6 & - & $0.91_{(7)}$ & $1.1_{(1)}$ & $2.00_{(3)}$ & 4.00 \\
\hline \multirow{3}{*}{$1476-3$} & \multirow{3}{*}{1150} & Arg & - & $0.3_{(2)}$ & - & $55.2(3)$ & - & $44.5_{(2)}$ & 100 & 3 & - & - & $0.94_{(1)}$ & $1.03_{(0)}$ & 1.97 \\
\hline & & Dol & - & - & $19_{(2)}$ & $31.9(5)$ & - & $48_{(1)}$ & 100 & 6 & - & $0.93_{(5)}$ & $0.99(1)$ & $2.05_{(3)}$ & 3.97 \\
\hline & & Mgs & - & $0.2_{(1)}$ & $43.5_{(5)}$ & $3.5_{(9)}$ & - & $52(1)$ & 100 & 3 & - & $0.92_{(2)}$ & $0.05_{(1)}$ & $1.01_{(1)}$ & 1.99 \\
\hline \multirow{2}{*}{ 1997-3 } & \multirow{2}{*}{1250} & Mgs & - & $0.23_{(1)}$ & $41.7_{(7)}$ & $6.8_{(6)}$ & - & $51_{(1)}$ & 100 & 3 & - & $0.89_{(2)}$ & $0.10_{(1)}$ & $1.00_{(2)}$ & 2.00 \\
\hline & & Dol & - & - & $23.1_{(4)}$ & $28_{(1)}$ & - & $48.9_{(8)}$ & 100 & 6 & - & $1.06_{(1)}$ & $0.87_{(3)}$ & $2.04_{(2)}$ & 3.97 \\
\hline \multirow{3}{*}{$2000-3$} & \multirow{3}{*}{1350} & Mgs & - & $0.3_{(2)}$ & $38.9_{(9)}$ & $8.5_{(9)}$ & - & $52.3_{(6)}$ & 100 & 3 & - & $0.83_{(3)}$ & $0.12_{(1)}$ & $1.02_{(1)}$ & 1.98 \\
\hline & & Dol & - & $0.21_{(5)}$ & $25.8(9)$ & $25.1_{(1)}$ & - & $48.9_{(9)}$ & 100 & 6 & - & $1.18_{(5)}$ & $0.77_{(0)}$ & $2.03_{(3)}$ & 3.97 \\
\hline & & Mgs & - & $0.09(9)$ & $40.3_{(3)}$ & $4.9_{(2)}$ & - & $54.6_{(3)}$ & 100 & 3 & - & $0.85_{(1)}$ & $0.07_{(0)}$ & $1.04_{(0)}$ & 1.97 \\
\hline \multirow[t]{2}{*}{$2001-3$} & \multirow[t]{2}{*}{1450} & Dol & - & - & $20_{(2)}$ & $30_{(1)}$ & - & $48.9_{(3)}$ & 100 & 3 & - & $0.95_{(6)}$ & 0.99 & $2.03_{(1)}$ & 3.97 \\
\hline & & $\mathrm{L}_{\text {carb-sil }}$ & $15_{(3)}$ & $0.4_{(3)}$ & $24_{(2)}$ & $21_{(4)}$ & $1.0_{(7)}$ & $38(4)$ & 100 & - & - & - & - & - & - \\
\hline $1811-3$ & 1550 & $\mathrm{~L}_{\text {carb-sil }}$ & $11_{(1)}$ & $0.5_{(3)}$ & $21_{(2)}$ & $17.3_{(1)}$ & $1.7_{(5)}$ & $48(2)$ & 100 & - & - & - & - & - & - \\
\hline \multicolumn{16}{|c|}{ Olivine-Ankerite-Pyrite System } \\
\hline \multirow{4}{*}{$932-6$} & \multirow{4}{*}{1050} & Dol $_{\text {core }}$ & - & $8_{(2)}$ & $17_{(2)}$ & $27.9_{(3)}$ & - & $47.3_{(5)}$ & 100 & 6 & $0.22_{(7)}$ & $0.78_{(7)}$ & $0.94_{(0)}$ & $2.01_{(1)}$ & 3.98 \\
\hline & & Dol mantle $_{\text {mat }}$ & - & $3(1)$ & $20.4_{(4)}$ & $27.6_{(3)}$ & - & $49.0_{(9)}$ & 100 & 6 & $0.08_{(3)}$ & $0.94_{(1)}$ & $0.9(2)$ & $2.02(1)$ & 3.98 \\
\hline & & $\mathrm{Dol}_{\text {rim }}$ & - & $0.6_{(1)}$ & $20.9(4)$ & $30.1_{(4)}$ & - & $48.5_{(4)}$ & 100 & 6 & $0.01_{(0)}$ & $0.96_{(2)}$ & $0.98_{(1)}$ & $2.01_{(0)}$ & 3.99 \\
\hline & & Mgs & - & $0.5_{(2)}$ & $42.3_{(5)}$ & $4_{(1)}$ & - & $53_{(2)}$ & 100 & 3 & $0.01_{(0)}$ & $0.90_{(2)}$ & $0.06_{(2)}$ & $1.00_{(0)}$ & 1.98 \\
\hline \multirow{2}{*}{$1476-6$} & \multirow{2}{*}{1150} & Dol & - & $0.4_{(1)}$ & $22.0_{(5)}$ & $28.2_{(2)}$ & - & $49.5_{(7)}$ & 100 & 6 & $0.01_{(1)}$ & $1.00_{(3)}$ & $0.91_{(1)}$ & $2.01_{(1)}$ & 3.97 \\
\hline & & Mgs & - & $0.5_{(2)}$ & $41.2_{(1)}$ & $4.8_{(4)}$ & - & $53.5_{(5)}$ & 100 & 3 & $0.01_{(0)}$ & $0.87_{(0)}$ & $0.07_{(0)}$ & $1.01_{(0)}$ & 1.97 \\
\hline \multirow{3}{*}{ 1997-6 } & \multirow{2}{*}{1250} & Dol & - & $0.4_{(2)}$ & $23.0_{(5)}$ & $27.1_{(7)}$ & - & $49.5_{(5)}$ & 100 & 6 & $0.01_{(0)}$ & $1.04_{(2)}$ & $0.88_{(2)}$ & $2.02_{(1)}$ & 3.98 \\
\hline & & Mgs & - & $1.4_{(0)}$ & $37.0_{(1)}$ & $7.49_{(9)}$ & - & $53.4_{(9)}$ & 100 & 3 & $0.01_{(0)}$ & $0.79_{(0)}$ & $0.11_{(0)}$ & $1.01_{(1)}$ & 1.97 \\
\hline & & Dol & - & $0.4_{(1)}$ & $26.0_{(3)}$ & $23.5_{(6)}$ & - & $50.2_{(2)}$ & 100 & 6 & $0.01_{(0)}$ & $1.16_{(1)}$ & $0.75_{(2)}$ & $2.01_{(1)}$ & 3.97 \\
\hline \multirow[t]{2}{*}{$2000-6$} & 1350 & Mgs & - & - & $38_{(2)}$ & $9_{(1)}$ & - & $52_{(1)}$ & 100 & 3 & - & $0.82_{(4)}$ & $0.13_{(2)}$ & $1.01_{(1)}$ & 1.99 \\
\hline & & $\mathrm{L}_{\text {carb }}$ & $2.7_{(1)}$ & $1.1_{(2)}$ & $16.6_{(7)}$ & $34.2_{(4)}$ & - & $45.4_{(7)}$ & 100 & - & - & - & - & - & - \\
\hline & & $\mathrm{L}_{\text {carb }}$ & $0.4_{(2)}$ & $3_{(1)}$ & $17_{(7)}$ & $25_{(3)}$ & - & $48_{(2)}$ & 100 & - & - & - & - & - & - \\
\hline $2001-6$ & 1450 & Mgs & - & $1.8_{(2)}$ & $42_{(1)}$ & $3.7_{(4)}$ & - & $52.4_{(9)}$ & 100 & 3 & $0.02_{(0)}$ & $0.89_{(3)}$ & $0.06_{(1)}$ & $1.00_{(1)}$ & 1.98 \\
\hline $1811-6$ & 1550 & $\mathrm{~L}_{\text {carb }}$ & $2_{(1)}$ & $5.0_{(5)}$ & $15_{(2)}$ & $16_{(1)}$ & - & $55_{(3)}$ & 100 & - & - & - & - & - & - \\
\hline
\end{tabular}

values in parentheses are one sigma errors of the means based on replicate electron microprobe analyses reported as least units cited; 4.3 (1) should be read as $4.3 \pm 0.1 \mathrm{wt}$. \%. 
Table 5. Averaged compositions of sulfides, as well as predominantly sulfur and sulfide melts.

\begin{tabular}{|c|c|c|c|c|c|c|c|c|c|c|c|c|c|c|}
\hline \multirow{2}{*}{ Run N } & \multirow{2}{*}{$\mathrm{T},{ }^{\circ} \mathrm{C}$} & \multirow{2}{*}{ Phase } & \multicolumn{9}{|c|}{ Mass Concentrations, wt. \% } & \multicolumn{3}{|c|}{ Cations Per Formula Unit } \\
\hline & & & $\mathrm{Fe}$ & $\mathrm{Mg}$ & Mn & $\mathrm{Ca}$ & $\mathbf{N i}$ & $\mathrm{S}$ & $\mathbf{O}$ & $\mathrm{C}$ & Total & $\mathrm{Fe}$ & $\mathrm{Ni}$ & $\mathrm{S}$ \\
\hline \multicolumn{15}{|c|}{ Olivine-Ankerite-Sulfur System } \\
\hline \multirow{2}{*}{$932-3$} & \multirow{2}{*}{1050} & Py & $45.0_{(5)}$ & - & - & - & $1.1_{(5)}$ & $53.6_{(5)}$ & - & - & 99.8 & $0.96_{(2)}$ & $0.02_{(1)}$ & 2 \\
\hline & & Ls & $1.5(6)$ & - & - & - & $0.4_{(2)}$ & $90.5_{(7)}$ & $7.5_{(9)}$ & - & 100 & - & - & - \\
\hline \multirow[b]{2}{*}{$1476-3$} & \multirow[b]{2}{*}{1150} & Py & $45.0_{(6)}$ & - & - & - & $1.1_{(6)}$ & $54.6_{(7)}$ & - & - & 99.8 & $0.94_{(1)}$ & $0.02_{(1)}$ & 2 \\
\hline & & Ls & $1.9(8)$ & - & - & - & $0.3_{(9)}^{(0)}$ & $89.5_{(4)}$ & $8.3_{(7)}$ & - & 100 & - & - & - \\
\hline \multirow[b]{2}{*}{$1997-3$} & \multirow[b]{2}{*}{1250} & $\mathrm{Py}$ & $45_{(1)}$ & - & - & - & $1.2(9)$ & $54_{(1)}$ & - & - & 99.7 & $0.96_{(1)}$ & $0.02_{(2)}$ & 2 \\
\hline & & Ls & $2.2_{(5)}$ & - & - & - & $0.4_{(1)}$ & $88.8_{(5)}$ & $8_{(1)}$ & - & 100 & - & - & - \\
\hline $2000-3$ & 1350 & Ls & $20.7_{(4)}$ & $0.2_{(2)}$ & $0.4_{(1)}$ & $0.4_{(1)}$ & $0.8_{(2)}$ & $67.1_{(5)}$ & $8_{(1)}$ & $2.4_{(5)}$ & 100 & - & - & - \\
\hline 2001-3 & 1450 & Ls & $22.6_{(8)}$ & $0.4_{(1)}$ & $0.3_{(1)}$ & $0.5_{(2)}$ & $0.7_{(2)}$ & $65_{(1)}$ & $7.6_{(8)}$ & $3.0_{(9)}$ & 100 & - & - & - \\
\hline $1811-3$ & 1550 & Ls & $22.5_{(1)}$ & $0.7_{(1)}$ & - & $0.4_{(1)}$ & $0.5_{(1)}$ & $53_{(2)}$ & $16_{(1)}$ & $6.3_{(5)}$ & 100 & - & - & - \\
\hline \multicolumn{15}{|c|}{ Olivine-Ankerite-Pyrite System } \\
\hline \multirow{2}{*}{$932-6$} & \multirow{2}{*}{1050} & $\mathrm{Po}$ & $59.4_{(9)}$ & - & - & - & $0.51_{(7)}$ & $39.7_{(8)}$ & - & - & 99.6 & $0.85_{(3)}$ & $0.01_{(0)}$ & 1 \\
\hline & & Py & $46.4_{(3)}$ & - & - & - & - & $53.2_{(3)}$ & - & - & 99.6 & $1.00_{(1)}$ & - & 2 \\
\hline \multirow[b]{2}{*}{$1476-6$} & \multirow{2}{*}{1150} & Po & $59.0_{(5)}$ & - & - & - & $0.4_{(1)}$ & $40.0_{(4)}$ & - & - & 99.5 & $0.84_{(1)}$ & $0.01_{(0)}$ & 1 \\
\hline & & $\mathrm{Py}$ & $46.1_{(1)}$ & - & - & - & - & $53.6_{(6)}^{(x)}$ & - & - & 99.7 & $0.98_{(0)}^{(1)}$ & - & 2 \\
\hline \multirow{2}{*}{$1997-6$} & \multirow{2}{*}{1250} & Po & $59.0_{(5)}$ & - & - & - & $0.5_{(2)}$ & $40.1_{(4)}$ & - & - & 99.6 & $0.84_{(1)}$ & $0.01_{(0)}$ & 1 \\
\hline & & $\mathrm{Py}$ & $45.7_{(4)}$ & - & - & - & - & $53.8_{(2)}^{(x)}$ & - & - & 99.5 & $0.97_{(1)}^{(1)}$ & - & 2 \\
\hline $2000-6$ & 1350 & $\mathrm{~L}_{\mathrm{Fe}-\mathrm{S}-\mathrm{O}}$ & $58.0_{(3)}$ & - & - & - & $0.7_{(2)}$ & $39.9_{(1)}$ & $1.1_{(1)}$ & - & 99.7 & - & - & - \\
\hline 2001-6 & 1450 & $\mathrm{~L}_{\mathrm{Fe}-\mathrm{S}-\mathrm{O}}$ & $58.9_{(2)}$ & - & - & - & $0.9_{(1)}$ & $38.9_{(2)}$ & $0.9_{(1)}$ & - & 99.5 & - & - & - \\
\hline $1811-6$ & 1550 & $\mathrm{~L}_{\mathrm{Fe}-\mathrm{S}-\mathrm{O}}$ & $61.3_{(3)}$ & - & $0.2_{(2)}$ & - & $0.9_{(1)}$ & $33.6_{(3)}$ & $3.4_{(3)}$ & - & 99.5 & - & - & - \\
\hline
\end{tabular}

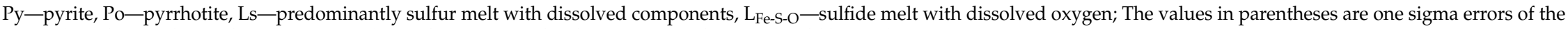
means based on replicate electron microprobe analyses reported as least units cited; $4.3_{(1)}$ should be read as $4.3 \pm 0.1 \mathrm{wt}$. \%. 


\subsection{Olivine-Ankerite-Pyrite System}

The experimental results are presented in Table 2. In relatively low-temperature experiments $\left(1050-1250^{\circ} \mathrm{C}, \mathrm{t}=60 \mathrm{~h}\right)$, the ankerite-olivine-pyrite interaction results in an assemblage of newly formed pyrrhotite, dolomite, magnesite, diopside, and graphite as well as recrystallized olivine and pyrite. The produced samples are polycrystalline aggregates of recrystallized olivine and sulfides, with interstices containing an aggregate of diopside, dolomite, magnesite, pyrrhotite, and pyrite (Figures $6 \mathrm{a}-\mathrm{c}$ and 7a). Recrystallized olivine (up to $300 \mu \mathrm{m}$ ) is characterized by zoning in the iron and nickel content (Figure 8). The highest iron content (up to $9.5 \mathrm{wt}$. \%) is found in the cores of large crystals, and the lowest iron content (up to almost pure forsterite) is observed in the rims of relatively large crystals and small interstitial crystals (Table 3). Clinopyroxene (diopside) is represented by relatively small crystals $(<20 \mu \mathrm{m})$ (Figure $6 \mathrm{~b}, \mathrm{c})$. The iron content of diopside varies in a range of $0.5-2.0 \mathrm{wt}$. \% and does not depend on temperature, as well as there is a deviation from the ideal stoichiometry in the form of predominance of magnesium over calcium. The Mg:Ca ratio in diopside depends on temperature and increases from 1.1 at $1050{ }^{\circ} \mathrm{C}$ to 1.21 at $1250{ }^{\circ} \mathrm{C}$.

Dolomite crystals $(10-100 \mu \mathrm{m})$ have a zoning in the iron content (Table 4). The highest iron content (up to $9.5 \mathrm{wt}$. \%) is found in medium and large crystals (about $100 \mu \mathrm{m}$ ); the lowest iron content (up to the complete absence of iron) is observed in the rims of medium and large crystals and in interstitial dolomite. As the temperature is increased, the zoning vanishes, and the stoichiometry is violated: the $(\mathrm{Mg}+\mathrm{Fe}) / \mathrm{Ca}$ ratio increases from 1.0 at 1050 to 1.18 at $1250{ }^{\circ} \mathrm{C}$. Magnesite is characterized by a size of up to $10 \mu \mathrm{m}$ and forms rims around diopside and sulfides. The iron content in magnesite varies within 0-2 wt. \%, regardless of the temperature; magnesite also contains calcium whose content increases from $4\left(1050^{\circ} \mathrm{C}\right)$ to $6.8 \mathrm{wt} . \%\left(1250^{\circ} \mathrm{C}\right)$. Pyrrhotite and pyrite form crystals of various sizes, from 5 to $300 \mu \mathrm{m}$, and occur in close intergrowth: pyrrhotite either overgrows pyrite along the margin, forming rims, or occurs inside pyrite grains and aggregates $\left(1250^{\circ} \mathrm{C}\right)$ (Figure $\left.6 \mathrm{c}\right)$. Furthermore, if pyrrhotite can present in the form of monomineral aggregates, pyrite always occurs in intergrowth with pyrrhotite. The composition of pyrite does not differ from the starting one. Pyrrhotite is characterized by a constant $\mathrm{Ni}$ content of about $0.7 \mathrm{wt}$ \%, regardless of the temperature. The $(\mathrm{Fe}+\mathrm{Ni}) / \mathrm{S}$ ratio in pyrrhotite varies from 0.81 to 0.86 . 

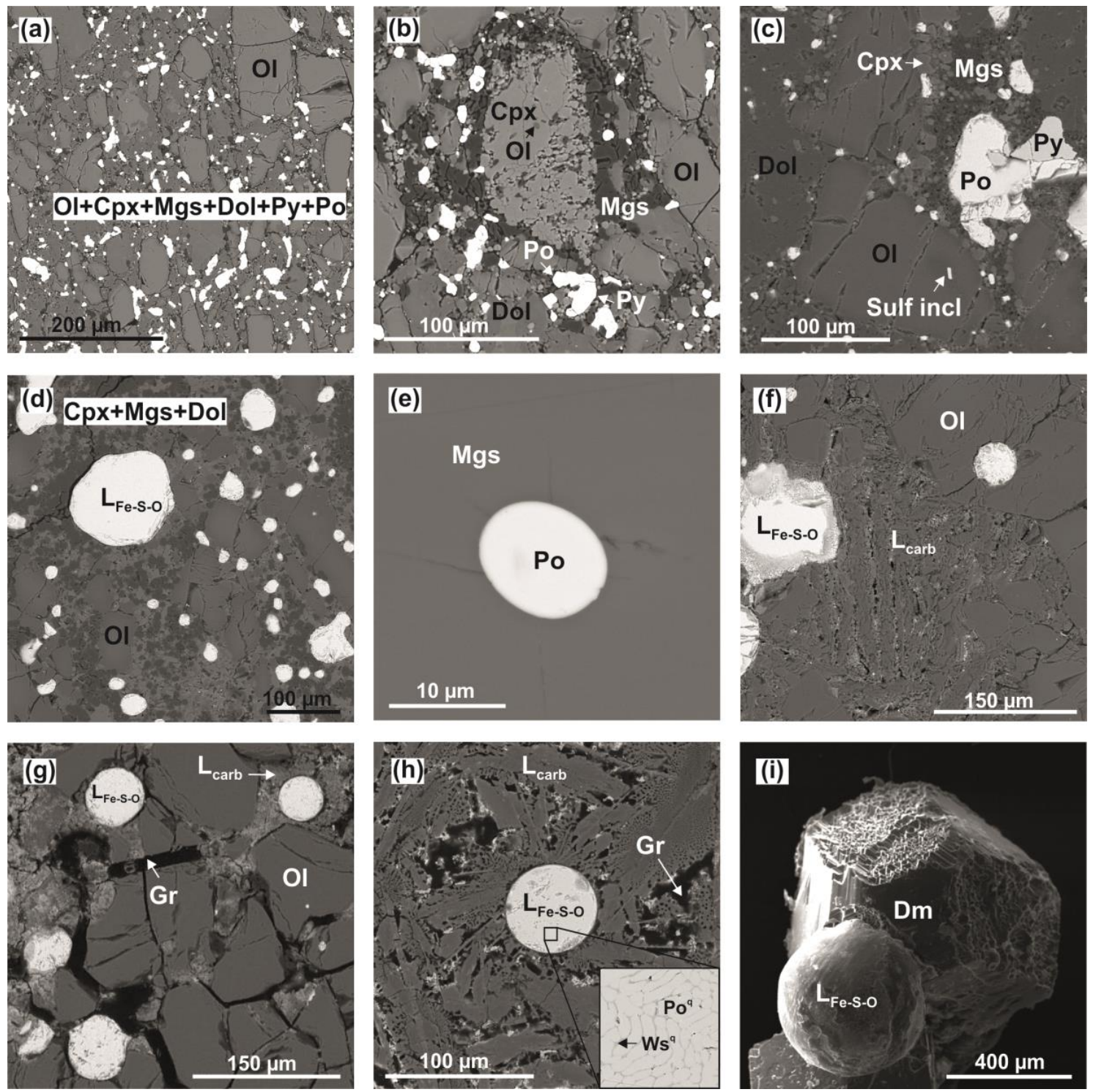

Figure 6. SEM micrographs of polished fragments of samples (a-h) and diamond seeds (i), which were obtained in the ankerite-olivine-pyrite system: $(\mathbf{a}, \mathbf{b})$-polycrystalline olivine aggregate with fine-crystalline olivine as well as newly formed clinopyroxene, magnesite, dolomite, pyrite, and pyrrhotite in interstices $\left(1050{ }^{\circ} \mathrm{C}\right)$; (c) - coarse-crystalline olivine and dolomite aggregate with fine-crystalline clinopyroxene, magnesite, pyrite, and pyrrhotite in interstices $\left(1250^{\circ} \mathrm{C}\right)$; $(\mathbf{d})-\mathrm{Fe}-\mathrm{S}-\mathrm{O}$ melt droplets in a polycrystalline aggregate of olivine, clinopyroxene, dolomite, and magnesite $\left(1350^{\circ} \mathrm{C}\right)$; (e) - pyrrhotite inclusion in magnesite $\left(1350^{\circ} \mathrm{C}\right)$; (f) - recrystallized olivine in a carbonate-silicate melt, as well as Fe-S-O melt droplets $\left(1450{ }^{\circ} \mathrm{C}\right) ;(\mathrm{g})$ - droplets of a quenched Fe-S-O melt in a polycrystalline aggregate of olivine and graphite $\left(1550^{\circ} \mathrm{C}\right) ;(\mathbf{h})$ —droplet of a quenched Fe-S-O melt in a carbonate-silicate melt $\left(1550{ }^{\circ} \mathrm{C}\right)$; (i) — diamond seed crystals with an overgrown layer and microspheres of a quenched Fe-S-O melt $\left(1550{ }^{\circ} \mathrm{C}\right)$; Ol—olivine, $\mathrm{Cpx}$ —clinopyroxene, Py—pyrite, Mgs—magnesite, Arg—aragonite,

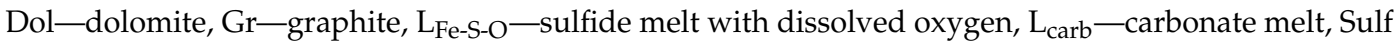
incl-sulfide inclusions in olivine;. 

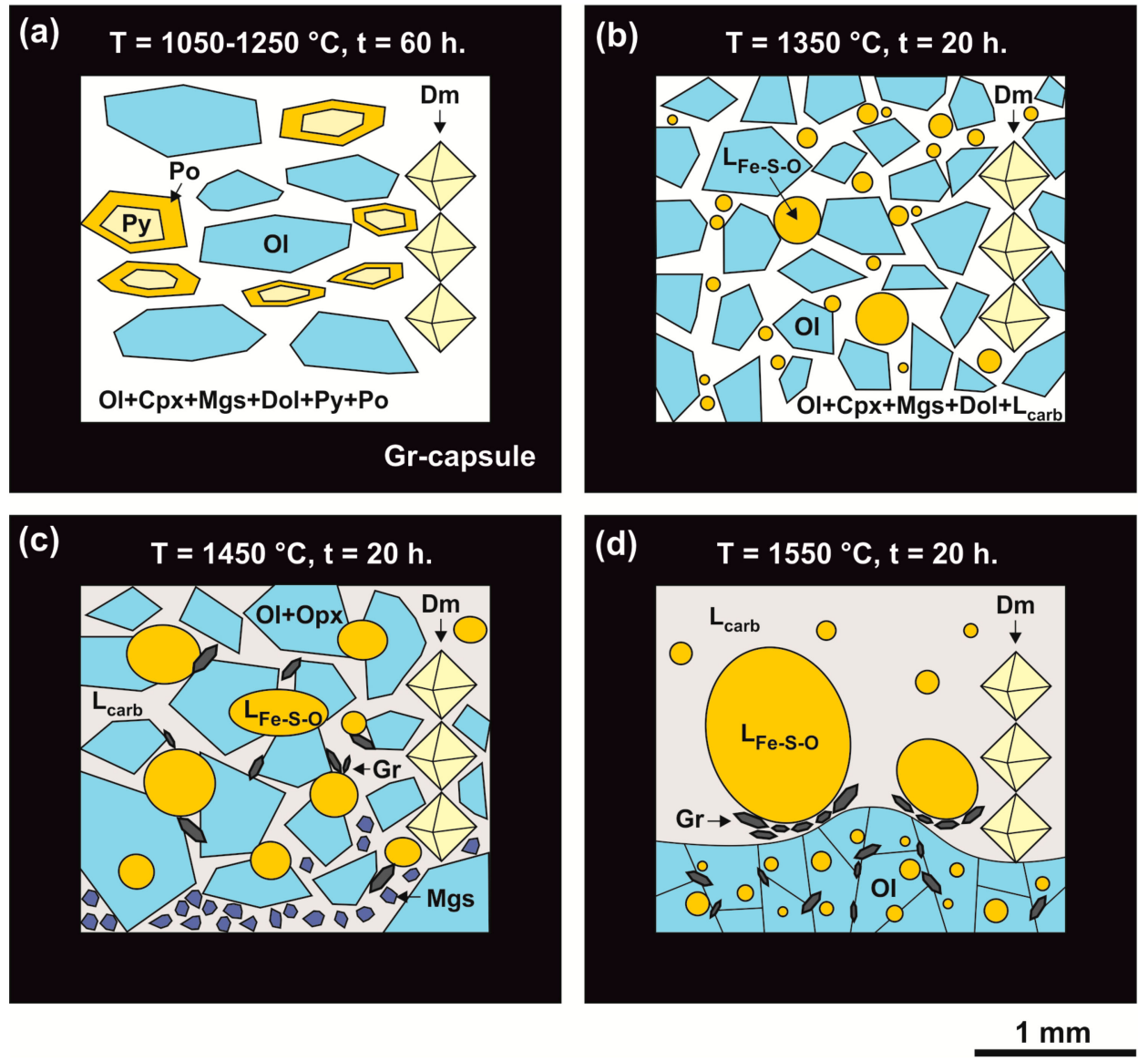

Figure 7. Schemes of samples produced in the olivine-ankerite-pyrite system at $6.3 \mathrm{GPa}$ in a temperature range of $1050-1550{ }^{\circ} \mathrm{C} ;(\mathbf{a}): \mathrm{T}=1050-1250{ }^{\circ} \mathrm{C} ;(\mathbf{b}): \quad \mathrm{T}=1350{ }^{\circ} \mathrm{C}$; (c): $\mathrm{T}$ $=1450{ }^{\circ} \mathrm{C} ;(\mathrm{d}): \mathrm{T}=1550{ }^{\circ} \mathrm{C}$; Po-pyrrhotite, Py-pyrite, Dol-dolomite, Mgs-magnesite,

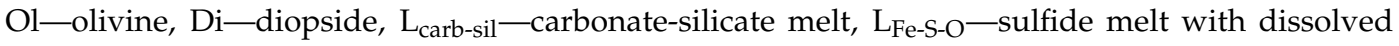
oxygen, Gr-graphite, Dm-diamond seed crystals.
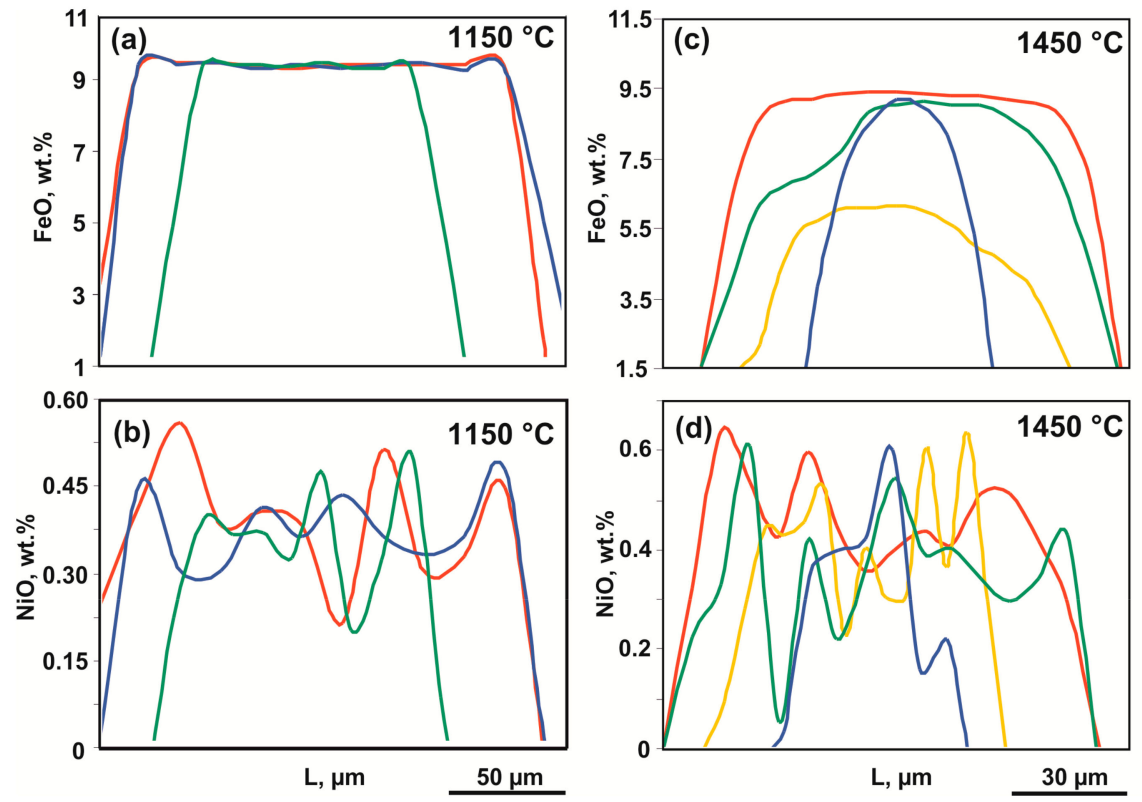

Figure 8. Representative profiles of the $\mathrm{FeO}$ and $\mathrm{NiO}$ distribution in recrystallized olivine (olivine-ankerite-pyrite system): (a,b) $-1150{ }^{\circ} \mathrm{C} ;(\mathbf{c}, \mathbf{d})-1350{ }^{\circ} \mathrm{C}$. 
At $1350{ }^{\circ} \mathrm{C}$, a polycrystalline aggregate of recrystallized olivine is formed, the interstices of which contain dolomite, magnesite, and diopside as well as a quenched carbonate melt and droplets of a quenched sulfide melt (Table 2; Figure 6d,e and Figure 7b). A quenching aggregate of the sulfide melt consists of pyrite, pyrrhotite, and wustite; the $(\mathrm{Fe}+\mathrm{Ni}) / \mathrm{S}$ atomic ratio in the aggregate is 0.83 , with $0.7 \mathrm{wt}$. \% of $\mathrm{Ni}$ and $1.0 \mathrm{wt}$. \% of $\mathrm{O}$. The carbonate melt is characterized by a Ca/Mg ratio of 1.5 and contains about 2.7 wt. $\%$ of $\mathrm{SiO}_{2}$ and 1 wt. \% of FeO. Olivine forms both large zoned crystals (up to $300 \mu \mathrm{m}$ ) with pyrrhotite inclusions and small ones (up to $15 \mu \mathrm{m}$ ) in interstices. Olivine contains $\sim 8 \mathrm{wt}$. \% of $\mathrm{FeO}$ in the core and $3 \mathrm{wt}$. \% of $\mathrm{FeO}$ in the rims. Newly formed diopside, dolomite, and magnesite contain $<1 \mathrm{wt}$. \% of $\mathrm{FeO}$.

At $1450{ }^{\circ} \mathrm{C}$, an assemblage of recrystallized olivine, magnesite, orthopyroxene, and graphite as well as carbonate and sulfide (with dissolved oxygen) melts was produced (Table 1). Figure 7c shows a schematic structure of the sample. Olivine forms a polycrystalline aggregate of large (up to $800 \mu \mathrm{m}$ ) zoned crystals (Figure $6 \mathrm{f} \%$ (core) to $3.8 \mathrm{wt}$. \% (rims) (Table 2). Magnesite and orthopyroxene $(10-15 \mu \mathrm{m})$ are present as rims around some olivine crystals. Magnesite is characterized by FeO and $\mathrm{CaO}$ contents of 1.8 wt. \% and 3.7 wt. \%, respectively; orthopyroxene contains $\mathrm{FeO}$ and $\mathrm{CaO}$ of 2 wt. \% and $3 \mathrm{wt}$. \%, respectively (Tables 3 and 4). The sulfide melt with dissolved oxygen is characterized by $\mathrm{a}(\mathrm{Fe}+\mathrm{Ni}) / \mathrm{S}$ ratio of 0.87 and contains $\sim 1 \mathrm{wt} . \%$ of $\mathrm{Ni}$ and $\mathrm{O}$ each (Table 5$)$. The carbonate melt is characterized by a $\mathrm{Ca} / \mathrm{Mg}$ atomic ratio of $\sim 0.95$ and a $\mathrm{FeO}$ concentration of $3 \mathrm{wt}$. \%.

At $1550{ }^{\circ} \mathrm{C}$, the formation of a solid-phase assemblage of olivine and graphite, growth of diamond on seed crystals, and the generation of carbonate and sulfide melts occur (Table 1; Figures $6 \mathrm{~g}-\mathrm{i}$ and $7 \mathrm{~d}$ ). Recrystallized zoned olivine (up to $500 \mu \mathrm{m}$ ) forms a polycrystalline aggregate in the lower part of the sample; olivine interstices contain graphite crystals (up to $150 \mu \mathrm{m}$ ) and droplets of a quenched sulfide melt. The FeO content in olivine varies from $6 \mathrm{wt}$. \% (core) to $0.4 \mathrm{wt}$. \% (rims); as at lower temperatures, olivine contains rounded sulfide inclusions. The sulfide melt forms rounded droplets (Figures $6 \mathrm{~g}-\mathrm{i}$ and $7 \mathrm{~d})$, is characterized by a $(\mathrm{Fe}+\mathrm{Ni}) / \mathrm{S}$ atomic ratio of about 1.05 , and contains $1 \mathrm{wt} . \%$ of $\mathrm{Ni}$ and $3.4 \mathrm{wt}$. \% of $\mathrm{O}$ (Table 5). The carbonate melt composition is characterized by a $\mathrm{Ca} / \mathrm{Mg}$ ratio of 0.76 and a $\mathrm{FeO}$ concentration of $5 \mathrm{wt}$. \%. Graphite crystals are present at the contact between the sulfide melt and the carbonate melt (Figure $7 \mathrm{~d}$ ), and quenched graphite is also present in a quenched carbonate melt (Figure 6h).

\subsection{Raman Spectral Characteristics of Produced Mineral Phases}

\subsubsection{Olivine, Orthopyroxene, and Clinopyroxene}

We used Raman spectroscopy to compare various zones of recrystallized olivine produced in the olivine-ankerite-sulfur system and the starting natural olivine (Lines (1)-(3) in Figure 9a). Comparison of the obtained spectra with each other as well as with the available literature data revealed the key indicative spectral characteristics of olivines exposed to reduced sulfur melts. The main Raman spectral characteristic of Fe,Mg-olivine is a doublet (DP) between $815 \mathrm{~cm}^{-1}$ and $860 \mathrm{~cm}^{-1}$. The emergence of DP is related to the activity of two stretching vibration modes of $\mathrm{SiO}_{4}$ tetrahedra ( $v 1$ and $\left.v 3\right)$. All active Raman modes of olivine may be divided into three spectral regions [48-50]. Peaks in the $150-400 \mathrm{~cm}^{-1}$ region are associated with lattice modes (translational and librational vibrations). Peaks in the $400-700 \mathrm{~cm}^{-1}$ region are associated with deformation vibrations of the $\mathrm{SiO}_{4}$ tetrahedron $(v 2$ and $\checkmark 4$ ) and displacements of Si and Mg. And peaks between $700 \mathrm{~cm}^{-1}$ and $1100 \mathrm{~cm}^{-1}$, including DP, are associated with internal stretching vibrations of the $\mathrm{SiO}_{4}$ tetrahedron $(v 1$ and $v 3)$. In the present study, all spectra of olivine (starting and recrystallized) exhibit the most intense doublet at 824 and $856 \mathrm{~cm}^{-1}$, peaks at 882, 926, and $966 \mathrm{~cm}^{-1}$, and also less intense peaks at $223-226,305,434,546,590$, and $613 \mathrm{~cm}^{-1}$. This set of peaks is characteristic of mantle olivines with the $\mathrm{Mg}_{1.8-2.0} \mathrm{Fe}_{0-0.2} \mathrm{SiO}_{4}$ composition [51]. The greatest similarity in a combination of peaks and in their relative intensities is found between the spectra of starting natural olivine and those of the cores of recrystallized olivine (Lines (2) and (3) in Figure 9a); in this case, the spectra of olivine rims, which underwent the most intense recrystallization, 
demonstrate several differences. A distinctive feature of the spectra of olivine rims is a set of peaks in low-frequency $\left(156,184\right.$, and $\left.206 \mathrm{~cm}^{-1}\right)$ and high-frequency $\left(1101 \mathrm{~cm}^{-1}\right)$ regions as well as a change in the relative intensities of DP peaks and a sharp decrease in the intensity of a peak at $926 \mathrm{~cm}^{-1}$. Peak intensity ratios for the doublet at $824 \mathrm{~cm}^{-1}$, associated with symmetric Si-O stretching vibrations, and $856 \mathrm{~cm}^{-1}$, associated with asymmetric Si-O stretching vibrations, are lowest $(\sim 0.60)$ in natural olivine and highest ( 1.2) in the rims of recrystallized olivine.
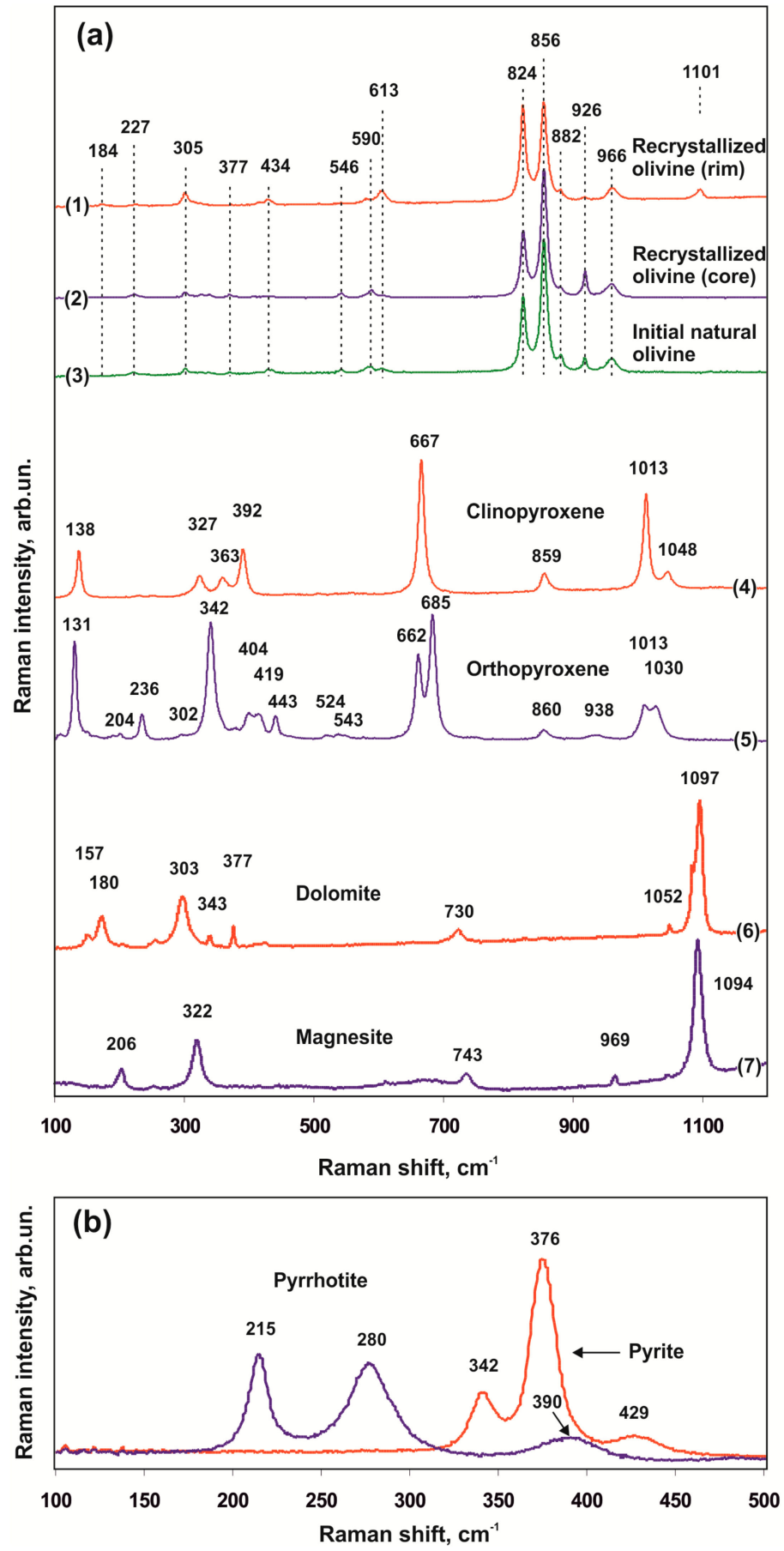

Figure 9. Representative Raman spectra (olivine-ankerite-sulfur system): (a)—recrystallized olivine $(1,2)$, starting natural olivine (3), newly formed phases-clinopyroxene (4), orthopyroxene (5), dolomite (6), and magnesite (7); (b)—sulfides in inclusions in olivine. 
Sulfide inclusions in olivine, which are formed in a temperature range of $1350-1550{ }^{\circ} \mathrm{C}$, are characterized by primarily spherical or rounded shapes. They are represented by quenched pyrrhotite or pentlandite with wide variations in the Ni content (2-19 wt. \%). Clinopyroxenes in polyphase inclusions within an assemblage with Ni-pyrrhotite contain $<<1 \mathrm{wt}$. \% of FeO. The spectra of Ni-pyrrhotite (Figure 9b) are characterized by broad lines at 215, 280, and $390 \mathrm{~cm}^{-1}$, and the spectra of pyrite are characterized by lines at 342,376 , and $429 \mathrm{~cm}^{-1}$. There are no consistent changes in the positions of these peaks, depending on the Ni concentration.

Representative spectra of Fe-poor and Fe-free clinopyroxenes and orthopyroxenes produced by recrystallization of olivine and ankerite in a sulfur melt are shown in lines (4) and (5) in Figure 9a. The composition of pyroxenes is characterized by the $\mathrm{FeO}$ concentration ranging from 0.05 to $1.5 \mathrm{wt}$. \%. Comparison of the Raman spectra of produced pyroxenes with the data of natural samples [52] demonstrated that a set of clinopyroxene peaks is most closely similar to the spectra of natural $\mathrm{En}_{0.50} \mathrm{Wo}_{0.50}$, and a set of orthopyroxene peaks is similar to the spectra of $\mathrm{En}_{0.975} \mathrm{Fs}_{0.025}$. The spectra of produced clinopyroxene lack peaks at 230,255 , and $558 \mathrm{~cm}^{-1}$, and the other peaks are shifted $1-5 \mathrm{~cm}^{-1}$ to the high-frequency region. The spectra of orthopyroxene lack a number of peaks characteristic of natural $\mathrm{En}_{0.975} \mathrm{Fs}_{0.025}: 552,583$, and $750 \mathrm{~cm}^{-1}$, and there is an additional peak at $384 \mathrm{~cm}^{-1}$. Most peaks in the spectra of produced orthopyroxene are shifted $1-6 \mathrm{~cm}^{-1}$ to the high-frequency region relative to those of natural orthopyroxene.

\subsubsection{Carbonates}

Raman spectra of newly formed magnesite and dolomite are shown in lines (6) and (7) in Figure 6b. Dolomite has two most intense peaks at $1097 \mathrm{~cm}^{-1}$ (symmetric stretching vibrations of $\mathrm{CO}_{3}$ groups, v1) and $303 \mathrm{~cm}^{-1}$ (lattice librational modes, L) as well as peaks at $730 \mathrm{~cm}^{-1}$ (deformation vibrations in the $\mathrm{O}-\mathrm{C}-\mathrm{O}$ plane, $v 4$ ) and $180 \mathrm{~cm}^{-1}$ (lattice translational modes, $\mathrm{T}$ ). All these Raman spectral characteristics are also detected in the spectra of natural dolomite. The Raman spectra of magnesite have the most intense peaks at 1094,743,322, and $206 \mathrm{~cm}^{-1}$, which are typical of natural magnesite and ferromagnesite $[53,54]$. In the spectra of produced magnesite and dolomite, there are peaks at $969 \mathrm{~cm}^{-1}$ and $1052 \mathrm{~cm}^{-1}$, which have not been previously described in studies of natural carbonates.

\subsubsection{Graphite}

Raman spectroscopy was used for comparative characterization and evaluation of structure defectiveness of large graphite crystals located at the contact of quenching aggregates of two immiscible melts and fine-grained quenching graphite (Figure 10a). Representative graphite spectra recorded in a range of $1000-3400 \mathrm{~cm}^{-1}$ are shown in Figure 10b. All graphite spectra have intense first-order bands D (1348-1352 $\left.\mathrm{cm}^{-1}\right), G\left(1579-1582 \mathrm{~cm}^{-1}\right)$, and D' $\left(1619-1623 \mathrm{~cm}^{-1}\right)$, as well as a second-order band $\mathrm{G}^{\prime}\left(2703-2719 \mathrm{~cm}^{-1}\right)$. Bands $\mathrm{G}$ and $\mathrm{D}$ are characterized by sharp narrow peaks. The most characteristic feature of quenching graphite produced in the olivine-ankerite-pyrite system is its enhanced imperfection as evidenced by an increased intensity of "defective" $\mathrm{D}$ and D' bands, with intensity ratios $\mathrm{I}_{\mathrm{D}} / \mathrm{I}_{\mathrm{G}} \sim 1.37$ and $\mathrm{I}_{\mathrm{D}^{\prime}} / \mathrm{I}_{\mathrm{G}} \sim 0.43$. In coarse-grained graphite formed in redox reactions, the $\mathrm{G}$ band intensity exceeds the $\mathrm{D}$ band intensity $\left(\mathrm{I}_{\mathrm{D}} / \mathrm{I}_{\mathrm{G}}=0.70\right)$; in this case, $\mathrm{I}_{\mathrm{D}^{\prime}} / \mathrm{I}_{\mathrm{G}}$ values also decrease to 0.20 . In large graphite crystals, the intensity of the second-order $G^{\prime}$ band increases as temperature is elevated (lines (1) and (2) in Figure 10b). 

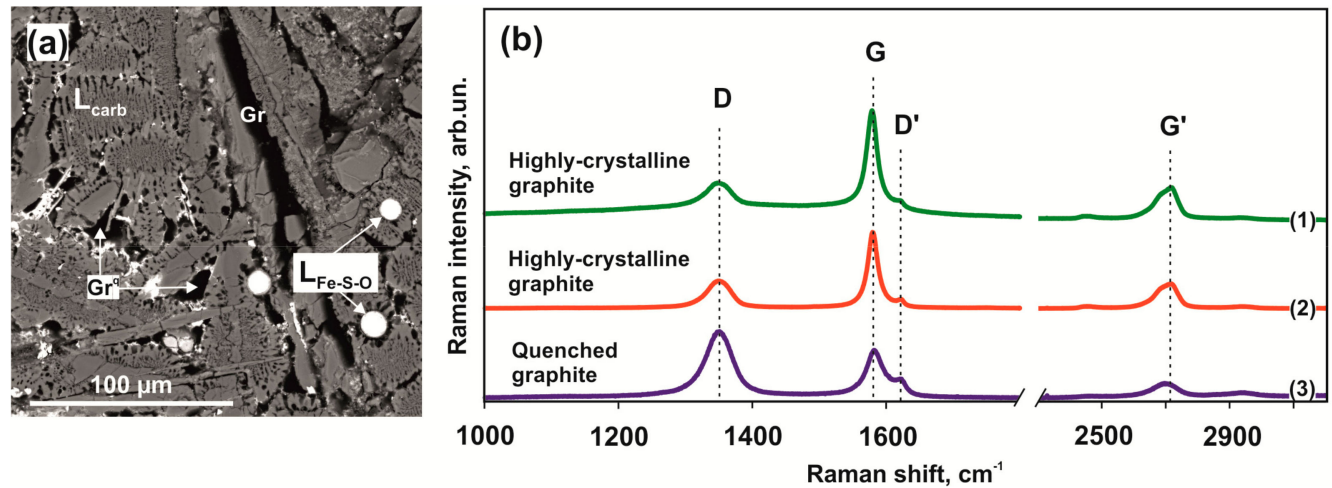

Figure 10. SEM micrograph of graphite in a quenched carbonate-silicate melt (a) and Raman spectra of graphite (b) obtained in the olivine-ankerite-pyrite system at $1550{ }^{\circ} \mathrm{C}(1)$ and (3) and $1450{ }^{\circ} \mathrm{C}(2)$.

\section{Discussion}

\subsection{Reconstruction of Interactions in the Olivine-Ankerite-Sulfur System}

An analysis of the experimental results revealed that at relatively low temperatures $\left(1050-1250{ }^{\circ} \mathrm{C}\right)$, sulfur occurs in a molten state, and pyrite is stable (Figure 4). At these temperatures, olivine recrystallizes in a sulfur melt. The main features of this recrystallization include: (i) an increase in the size of olivine; (ii) a zonal structure of crystals, with a Fe-rich core and a Mg-rich (Ni-free and Fe-free) rim; (iii) formation of pyrite and diopside inclusions in Fe-and Ni-free olivine; (iv) some features in the Raman spectral characteristics of olivine (Figure 9a). A similar decrease in the concentrations of $\mathrm{FeO}$ and $\mathrm{NiO}$ and an increase in the $\mathrm{MgO}$ content from the core to rims of olivine crystals are considered as the main feature of olivine sulfidation in nature [29]. We found that recrystallization is accompanied by partial extraction of $\mathrm{Fe}, \mathrm{Ni}$, and other elements $(\mathrm{Si}, \mathrm{Mg}$, and $\mathrm{O}$ ) from olivine to a sulfur melt. Simultaneously with this process in the sulfur melt, there is recrystallization of Fe-bearing carbonate (ankerite), followed by extraction of $\mathrm{Fe}, \mathrm{Ca}, \mathrm{Mg}, \mathrm{C}$, and $\mathrm{O}$ into the sulfur melt. Therefore, recrystallization of $\mathrm{Fe}$,Ni-olivine and ankerite in the sulfur melt with dissolved components leads to crystallization of newly formed phases including pyrite and almost Fe-free carbonates-magnesite, dolomite, and aragonite $(\leq 0.3$ wt. \% of $\mathrm{FeO})$, and diopside; the contents of iron and nickel in recrystallized olivine decrease. It is important to note that there was no partial melting of olivine and carbonate in the entire temperature range $\left(1050-1250{ }^{\circ} \mathrm{C}\right)$, which was previously thought $[28,30]$ to be a necessary condition for sulfidation reactions.

One of the most informative results in terms of reconstruction of sulfidation processes is the found alternation of reaction zones around recrystallized olivine. Investigation of these zonal aggregates revealed the order of phase formation during the interaction of olivine and ankerite with a sulfur melt: an assemblage of olivine depleted in iron and nickel, diopside, magnesite, and pyrite is formed at the initial stage of sulfidation, and a sub-equilibrium assemblage of Ni-bearing pyrite with iron-free diopside and aragonite is formed at the final stage. Therefore, upon completion of the sulfidation reactions of $\mathrm{Fe}$, Ni-olivine and ankerite in a range of $1050-1250^{\circ} \mathrm{C}$, sulfide becomes the only phase-concentrator of iron and nickel, while clinopyroxene contains magnesium, calcium, and silicon, and carbonate comprises carbon and calcium.

At relatively high temperatures $\left(\geq 1350^{\circ} \mathrm{C}\right)$, newly formed pyrite is unstable; for this reason, solid-phase sulfide is absent in samples, and major portions of iron and nickel occur in a sulfur melt. Under these conditions, olivine and ankerite recrystallize in the sulfur melt, which is accompanied by almost complete extraction of metals ( $\mathrm{Fe}, \mathrm{Ni}, \mathrm{Mg}, \mathrm{Ca}, \mathrm{Si})$, carbon, and oxygen into the melt, followed by crystallization of newly formed magnesite, dolomite, and diopside $\left(1350^{\circ} \mathrm{C}\right)$ or enstatite $\left(1450{ }^{\circ} \mathrm{C}\right)$. Only small amounts of $\mathrm{Mg}, \mathrm{Ca}, \mathrm{Si}$, and $\mathrm{C}$ are preserved in the sulfur melt due to crystallization of carbonates and silicates, but the melt remains enriched in Fe and Ni (Fe-S, Ni-S ligands- "sulfide component"). At temperatures above $1350{ }^{\circ} \mathrm{C}$, there is partial melting of dolomite and diopside with 
the formation of a high-calcium carbonate-silicate melt. Under these conditions, the formation of large graphite crystals $\left(1350-1550^{\circ} \mathrm{C}\right.$ ) and diamond growth (at $1550{ }^{\circ} \mathrm{C}$ ) on seed crystals occur at the contact of two immiscible melts. The most likely mechanism of graphite formation and diamond growth is the redox interaction between a reduced sulfur melt (with dissolved $\mathrm{Fe}$ and $\mathrm{Ni}$, as "sulfide component") and an oxidized carbonate-silicate melt. A similar mechanism for the formation of graphite and diamond upon reduction of carbonate, carbonate melt, or $\mathrm{CO}_{2}$-fluid by sulfides was demonstrated earlier in $[21,55,56]$.

It should be noted that at the highest temperatures $\left(1450\right.$ and $\left.1550{ }^{\circ} \mathrm{C}\right)$, during olivine recrystallization in the sulfur melt, rounded inclusions of a Ni-enriched (up to $\sim 19 \mathrm{wt}$. \%) sulfide melt are formed in olivine crystals. The most likely mechanism for the formation of these inclusions during the olivine-sulfur interaction is capture of sulfide droplets formed upon segregation according to the scheme $x \mathrm{FeO}_{\mathrm{ol}}+\mathrm{yNiO}$ ol $+\mathrm{S}_{\text {melt }} \rightarrow \mathrm{Fe}_{\mathrm{x}-\mathrm{z}} \mathrm{O}_{\mathrm{x}+\mathrm{y}} \mathrm{S}_{\text {melt }}+\mathrm{Ni}_{\mathrm{y}} \mathrm{Fe}_{\mathrm{z}} \mathrm{S}_{\text {melt }}$, which is similar to that proposed in [57].

\subsection{Reconstruction of Interactions in the Olivine-Ankerite-Pyrite System}

In this system, in a range of $1050-1250{ }^{\circ} \mathrm{C}$, pyrite is the source of a small amount of sulfur fluid, the presence of which provides conditions for recrystallization of mineral phases as well as transport of dissolved components in the reaction volume. Like in the olivine-ankerite-sulfur system, the main phase formation processes under these conditions are olivine and ankerite recrystallization accompanied by extraction of metals ( $\mathrm{Fe}$ and $\mathrm{Ni}$ ) into the fluid and the formation of pyrrhotite. Sulfidation of olivine and ankerite at relatively low temperatures leads to the crystallization of newly formed clinopyroxene, dolomite, magnesite, and pyrrhotite as well as to recrystallization of starting olivine with a significant change in its composition. At temperatures above $1250{ }^{\circ} \mathrm{C}$, partial melting of both sulfide and carbonate phases occurs in the system; in this case, the bulk composition of produced melts is quite close to the stoichiometry of pyrrhotite and dolomite, respectively. An increase in the temperature of experiments in a range of $1350-1550{ }^{\circ} \mathrm{C}$ and a corresponding increase in the degree of melting lead to a change in the solid-phase assemblages $\mathrm{Ol}_{\text {recryst }}+\mathrm{Cpx}+\mathrm{Dol}+\mathrm{Mgs} \rightarrow \mathrm{Ol}_{\text {recryst }}+\mathrm{Opx}$ $+\mathrm{Mgs} \rightarrow \mathrm{Ol}_{\text {recryst }}$. The lack of Ca-enriched mineral phases at 1450 and $1550{ }^{\circ} \mathrm{C}$ directly correlates with the specificity of a high-calcium carbonate melt composition, behavior of calcium as an incompatible element, and Ca extraction into a newly formed carbonate melt. It should also be noted that the carbonate melt produced at $1550{ }^{\circ} \mathrm{C}$ is characterized by lower concentrations of iron and calcium and increased concentrations of magnesium relative to those in the melt formed at $1450{ }^{\circ} \mathrm{C}$, which may be explained by complete melting of carbonates.

In particular, we would like to address the issue of elemental carbon phase formation. At temperatures above $1350{ }^{\circ} \mathrm{C}$, the olivine-ankerite-pyrite interaction leads to the formation of coarse-grained graphite $\left(1450\right.$ and $\left.1550{ }^{\circ} \mathrm{C}\right)$ and diamond growth $\left(1550{ }^{\circ} \mathrm{C}\right)$ on seed crystals at the contact of sulfide and carbonate melts. As in the olivine-ankerite system, the most likely mechanism of graphite formation and diamond growth is the redox interaction between a reduced sulfide melt and an oxidized carbonate melt. Involvement of this mechanism is particularly evidenced by the observed oxidation of a sulfide melt and an increase in the concentration of oxygen dissolved in it. A similar change in the sulfide melt composition during reduction of carbonate to graphite or diamond was previously demonstrated in $[55,56]$.

\subsection{Raman Spectral Characteristics and Indicative Properties of Mineral Phases Produced in Sulfidation Processes}

\subsubsection{Olivine}

It is found that the most typical indicative features of sulfidized olivine are as follows: (i) no nickel impurity and a low-iron content in olivine; (ii) a zonal structure of the crystals, with an iron-rich core and a magnesium rim; (iii) the presence of $\mathrm{Fe}, \mathrm{Ni}$-sulfide inclusions as well as polyphase sulfide + diopside 
inclusions in low-iron or iron-free olivine; (iv) some features of the Raman spectral characteristics of olivine. These indicative characteristics imply recrystallization of olivine in a sulfur melt, extraction of $\mathrm{Fe}$ and $\mathrm{Ni}$ into the melt with a subsequent decrease in the concentrations of $\mathrm{FeO}$ and $\mathrm{NiO}$ in olivine, and formation of newly formed sulfides and pyroxenes.

Comparison of the spectra of starting and recrystallized olivine with each other as well as with the available literature data revealed the key indicative spectral characteristics of sulfidized olivine. An analysis of the published literature data showed that peaks at 206 and $1101 \mathrm{~cm}^{-1}$, which were present in the spectra of recrystallized olivine, had not previously been described in the spectra of natural olivine [51,58-63]. Previously, a study [51] demonstrated that a decrease in the $926 \mathrm{~cm}^{-1}$ peak intensity for forsterite $\left(\mathrm{Fo}_{100}\right)$ and a forsterite-fayalite solid solution $\left(\mathrm{Fo}_{90} \mathrm{Fa}_{10}\right)$, similar to that observed in this study, may be due to an increase in the temperature. Therefore, additional peaks at 206 and $1101 \mathrm{~cm}^{-1}$ as well as a change in relative intensities of some peaks may be considered as indicative Raman spectral characteristics of olivines subjected to metasomatic effects of reduced sulfur melts or fluids.

\subsubsection{Carbonates}

It was found that the indicative characteristics of dolomite and magnesite involved in the sulfidation processes are the presence of sulfide melt inclusions in them as well as some features of Raman spectra. The experimental results demonstrated that capture of sulfide melt inclusions occurred upon carbonate recrystallization during the interaction between ankerite and a reduced sulfur melt. During recrystallization, iron and carbon were extracted from carbonate, followed by the formation of a sulfide melt, graphite, and Fe-poor carbonates-magnesite and dolomite \pm aragonite. An additional peak in the spectra of produced magnesite- $-969 \mathrm{~cm}^{-1}$, and $1052 \mathrm{~cm}^{-1}$ for dolomite, not previously described for natural carbonates, may be considered as the Raman spectral indicative characteristics of these carbonates formed due to sulfidation of the carbonate matrix by reduced sulfur melt.

\subsubsection{Graphite}

Raman spectroscopy was used for comparative characterization and assessment of structure defectiveness of graphite produced (i) in redox reactions between a carbonate melt and a sulfur melt with dissolved Fe and $\mathrm{Ni}$ and (ii) upon quenching of a carbon-saturated carbonate melt. The most characteristic feature of quenching graphite produced in the olivine-ankerite-pyrite system is its enhanced defectiveness, which is evidenced by an increased intensity of “defective" D and D' bands, with intensity ratios $\mathrm{I}_{\mathrm{D}} / \mathrm{I}_{\mathrm{G}^{\prime}} \sim 1.37$ and $\mathrm{I}_{\mathrm{D}^{\prime}} / \mathrm{I}_{\mathrm{G}} \sim 0.43$, respectively. In large graphite crystals formed in redox reactions, the $G$ band intensity exceeds that of the $\mathrm{D}$ band $\left(\mathrm{I}_{\mathrm{D}} / \mathrm{I}_{\mathrm{G}}=0.70\right)$; in this case, the $\mathrm{I}_{\mathrm{D}^{\prime}} / \mathrm{I}_{\mathrm{G}}$ values also decrease to 0.20 . In coarse-grained graphite, the second-order $G^{\prime}$ band intensity increases as the temperature is raised (lines (1) and (2) in Figure 10a). In general, the Raman spectroscopy data of graphite crystals produced by redox interactions demonstrated a low degree of their defectiveness.

\subsection{Scenarios of Metasomatic Interactions of Reduced Sulfur-Rich Fluid with Olivine and Carbonates in the Earth's Upper Mantle}

The studied olivine-ankerite-sulfur and olivine-ankerite-pyrite interactions may be considered as the basis for modeling of metasomatic processes under subduction conditions with involvement of reduced S-bearing melts/fluids as well as oxidized carbonate and carbonate-silicate melts. As experimentally found [5], fluid transport of sulfur from the oceanic crust to the mantle wedge rocks can occur in a wide range of $f_{2}$ values, which confirms the possibility of interactions between mantle rocks and melts/fluids containing sulfur in a reduced state. It was demonstrated earlier [4] that sulfur may be added to deeper parts of the subarc mantle via pyrite breakdown, causing sulfur fluid/melt release. Our findings indicate that recrystallization of Fe,Ni-olivine and Fe-bearing carbonate and their sulfidation may occur in the presence of even a small amount of reduced S-bearing melt/fluid ( \pm sulfide melt) at relatively low temperatures on the boundary between the "cold" slab and the mantle wedge. Therefore, one of the most important results of our study is identification of the conditions necessary for the olivine 
and carbonate sulfidation reactions in natural environments, under subduction settings. Partial melting of olivine and carbonate was experimentally demonstrated not to be a mandatory aspect for extraction of metals and formation of sulfides; in addition, this mechanism of sulfide formation may operate at moderately oxidizing conditions, not only at sharply reducing conditions, as previously thought. It was experimentally demonstrated that subduction of sulfur-bearing minerals and carbonates to ultrabasic rocks, to the upper mantle depths, can be accompanied by the generation of metasomatic agents, both reducing (sulfur fluids or sulfide melts) and oxidizing (carbonate or carbonate-silicate melts). These metasomatic agents are not only redox-contrasted but also immiscible $[64,65]$. It should be noted that P,T-parameters for the formation of these agents directly depend on the composition of subducted minerals, host rocks, presence of other fluids or melts, and fugacity of oxygen and sulfur. In a relatively low-temperature stage of metasomatism on the boundary between the "cold" slab and the mantle wedge, mantle silicate rocks and iron-bearing slab carbonates were experimentally demonstrated to be reworked by mobile sulfur fluids. This metasomatic reworking leads to extraction of metals, carbon, and oxygen from olivine and Fe-carbonates, followed by the formation of sulfides as well as crystallization of iron-free silicates and carbonates with inclusions of Fe,Ni-sulfides. A further, more intensive (more high-temperature) metasomatic interaction of these rocks with reduced sulfur metasomatic agents containing dissolved components leads to significant enrichment of S-bearing fluids/melts with sulfide, reduction in their mobility, and formation of sulfide cumulates. The experimentally proven mechanism for the formation of mantle sulfides and sulfide melts by the olivine sulfidation reaction may be considered as one of the causes for the formation of Ni-sulfide inclusions in forsterite crystals $[15,19,66]$. Upon the generation of two metasomatic agents, reduced (sulfur fluid or sulfide melt) and oxidized (carbonate or carbonate-silicate melts) ones, which can occur in a rather wide range of temperatures and pressures in the undergoing slab, graphite- and diamond-producing redox reactions take place. Our findings are in good agreement with the results of experimental studies [21,55,56]: the formation of graphite and diamond from carbonate carbon under upper mantle conditions may occur upon reduction of carbonates or carbonate melts by sulfide melts.

\section{Conclusions}

It is found that during the olivine-ankerite-sulfur and olivine-ankerite-pyrite interactions at pressure of $6.3 \mathrm{GPa}$ and temperatures from 1050 to $1250{ }^{\circ} \mathrm{C}$, sulfidation of Fe,Ni-olivine and ankerite occurs due to recrystallization of silicate and carbonate phases in a sulfur melt/fluid, extraction of metals, carbon, and oxygen into the melt, and subsequent crystallization of sulfides and iron-free phases-diopside and alkaline earth carbonates. At temperatures above $1350{ }^{\circ} \mathrm{C}$, two immiscible, oxidized carbonate and reduced sulfide, melts are generated, and their redox interaction leads to graphite formation and diamond growth.

It is experimentally demonstrated that the main features of metasomatic alteration of Fe,Ni-bearing olivine by reduced sulfur fluids/melts include: (i) a zonal structure of olivine crystals, with an iron-rich core and magnesium-rich (nickel-free and iron-free) rims; (ii) the formation of pyrite and pyrrhotite ( \pm pyroxene) inclusions in almost iron-free and nickel-free olivine; (iii) some features of the Raman spectral characteristics of olivine (peaks at 206 and $1101 \mathrm{~cm}^{-1}$ ).

It was experimentally shown that metasomatic processes involving S- and C-bearing minerals, which may potentially occur in the upper mantle are accompanied by the generation of sulfide and carbonate melts, extraction of metals, carbon, and oxygen from silicates and carbonates into reduced sulfur fluid, as well as by graphite formation and diamond growth from carbon of carbonates.

Author Contributions: Conceptualization, Y.B. (Yuliya Bataleva) and Y.P.; Data curation, Y.B. (Yuliya Bataleva) and Y.P.; Formal analysis, E.Z., I.N. and Y.B. (Yuliya Bataleva); Investigation, E.Z., I.N. and Y.B. (Yuliya Bataleva); Methodology, E.Z., I.N. and Y.B. (Yuri Borzdov); Visualization, Y.B. (Yuliya Bataleva); Writing-original draft, E.Z., I.N. and Y.B. (Yuliya Bataleva); Writing-review and editing, Y.B. (Yuliya Bataleva) and Y.P.

Funding: This research was performed by state assignment of IGM SB RAS (0330-2016-0007).

Acknowledgments: The authors thank A. Moskalev for his assistance in the work preparation. 
Conflicts of Interest: The authors declare no conflict of interest.

\section{References}

1. Alt, J.; Shanks, W.C.; Jackson, M.C. Cycling of sulfur in subduction zones: The geochemistry of sulfur in the Mariana Island Arc and back-arc trough. Earth Planet. Sci. Lett. 1993, 119, 477-494. [CrossRef]

2. Evans, K.A. The redox budget of subduction zones. Earth Sci. Rev. 2012, 113, 11-32. [CrossRef]

3. Evans, K.A.; Powell, R. The effect of subduction on the sulphur, carbon and redox budget of lithospheric mantle. J. Metamorph. Geol. 2015, 33, 649-670. [CrossRef]

4. Tomkins, A.G.; Evans, K.A. Separate zones of sulfate and sulfide release from subducted mafic oceanic crust. Earth Planet. Sci. Lett. 2015, 428, 73-83. [CrossRef]

5. Jégo, S.; Dasgupta, R. The fate of sulfur during fluid-present melting of subducting basaltic crust at variable oxygen fugacity. J. Petrol. 2014, 55, 1019-1050. [CrossRef]

6. Labidi, J.; Cartigny, P.; Jackson, M.G. Multiple sulfur isotope composition of oxidized Samoan melts and the implications of a sulfur isotope 'mantle array' in chemical geodynamics. Earth Planet. Sci. Lett. 2015, 417, 28-39. [CrossRef]

7. Wallace, P.J.; Edmonds, M. The sulfur budget in magmas: Evidence from melt inclusions, submarine glasses, and volcanic gas emissions. Rev. Mineral. Geochem. 2011, 73, 215-246. [CrossRef]

8. Shirey, S.B.; Cartigny, P.; Frost, D.G.; Keshav, S.; Nestola, F.; Nimis, P.; Pearson, D.G.; Sobolev, N.V.; Walter, M.J. Diamonds and the geology of mantle carbon. Rev. Mineral. Geochem. 2013, 75, 355-421. [CrossRef]

9. Oganov, A.R.; Hemley, R.J.; Hazen, R.M.; Jones, A.P. Structure, bonding, and mineralogy of carbon at extreme conditions. Rev. Mineral. Geochem. 2013, 75, 47-77. [CrossRef]

10. Bulanova, G.P. The formation of diamond. J. Geochem. Explor. 1995, 53, 1-23. [CrossRef]

11. Wang, A.; Pasteris, J.D.; Meyer, H.O.A.; DeleDuboi, M.L. Magnesite-bearing inclusion assemblage in natural diamond. Earth Planet. Sci. Lett. 1996, 141, 293-306. [CrossRef]

12. Navon, O.; Hutcheon, I.D.; Rossman, G.R.; Wasserburg, G.J. Mantle-derived fluids in diamond micro-inclusions. Nature 1988, 335, 784-789. [CrossRef]

13. Schrauder, M.; Navon, O. Hydrous and carbonatitic mantle fluids in fibrous diamonds from Jwaneng, Botswana. Geochim. Cosmochim. Acta 1994, 58, 761-771. [CrossRef]

14. Sobolev, N.V.; Kaminsky, F.V.; Griffin, W.L.; Yefimova, E.S.; Win, T.T.; Ryan, C.G.; Botkunov, A.I. Mineral inclusions in diamonds from the Sputnik kimberlite pipe, Yakutia. Lithos 1997, 39, 135-157. [CrossRef]

15. Lorand, J.P.; Gregoire, M. Petrogenesis of base metal sulphide assemblages of some peridotites from the Kaapvaal craton (South Africa). Contrib. Mineral. Petrol. 2006, 151, 521-538. [CrossRef]

16. Stachel, T.; Harris, J.W. The origin of cratonic diamonds-Constraints from mineral inclusions. Ore Geol. Rev. 2008, 34, 5-32. [CrossRef]

17. Leung, I.S.; Kuo, W.X. Silicon carbide cluster entrapped in a diamond from Fuxian, China. Am. Mineral. 1990, 75, 1110-1119.

18. Giuliani, A.; Fitzpayne, A.; Phillips, D.; Hergt, J.; Woodhead, J.D.; James Farquhar, J.; Fiorentini, M.L.; Russell, N.; Drysdale, R.N.; Wu, N. Mantle oddities: A sulphate fluid preserved in a MARID xenolith from the Bultfontein kimberlite (Kimberley, South Africa). Earth Planet. Sci. Lett. 2013, 376, 74-86. [CrossRef]

19. Andersen, T.; Neumann, E.R. Fluid inclusions in mantle xenoliths. Lithos 2001, 55, 301-320. [CrossRef]

20. Newton, R.C.; Manning, C.E. Solubility of anhydrite, $\mathrm{CaSO} 4$, in $\mathrm{NaCl}-\mathrm{H}_{2} \mathrm{O}$ solutions at high pressures and temperatures: Applications to fluid-rock interaction. J. Petrol. 2005, 46, 701-716. [CrossRef]

21. Palyanov, Y.N.; Borzdov, Y.M.; Bataleva, Y.V.; Sokol, A.G.; Palyanova, G.A.; Kupriyanov, I.N. Reducing role of sulfides and diamond formation in the Earth's mantle. Earth Planet. Sci. Lett. 2007, 260, 242-256. [CrossRef]

22. Carroll, M.R.; Rutherford, M.J. Sulfide and sulfate saturation in hydrous silicate melts. J. Geophys. Res. 1985, 90, 601-612. [CrossRef]

23. Lorand, J.P.; Alard, O.; Godard, M. Highly siderophile elements and the multi-stage metasomatic history of Kerguelen lithospheric mantle (south Indian Ocean). Chem. Geol. 2004, 208, 195-215. [CrossRef]

24. Zajacz, Z.; Candela, P.A.; Piccoli, P.M.; Sanchez-Valle, C.; Waelle, M. Solubility and partitioning behavior of $\mathrm{Au}, \mathrm{Cu}, \mathrm{Ag}$ and reduced $\mathrm{S}$ in magmas. Geochim. Cosmochim. Acta 2013, 112, 288-304. [CrossRef] 
25. Alard, O.; Lorand, J.-P.; Reisberg, L.; Bodinier, J.-L.; Dautria, J.-M.; O'Reilly, S. Volatile-rich metasomatism in Montferrier xenoliths (Southern France): Implications for the abundances of chalcophile and highly siderophile elements in the subcontinental mantle. J. Petrol. 2011, 52, 2009-2045. [CrossRef]

26. Delpech, G.; Lorand, J.P.; Grégoire, M.; Cottin, J.-Y.; O’Reilly, S.Y. In-situ geochemistry of sulfides in highly metasomatized mantle xenoliths from Kerguelen, southern Indian Ocean. Lithos 2012, 154, $296-314$. [CrossRef]

27. Eggler, D.H.; Lorand, J.P. Mantle sulfide geobarometry. Geochim. Cosmochim. Acta 1993, 57, $2213-2222$. [CrossRef]

28. Fleet, M.E.; MacRae, N.D. Sulfidation of Mg-rich olivine and the stability of niningerite in enstatite chondrites. Geochim. Cosmochim. Acta 1987, 51, 1511-1521. [CrossRef]

29. Papike, J.J.; Spilde, M.N.; Fowler, G.W.; Layne, G.D.; Shearer, C.K. The Lodran primitive achondrite: Petrogenetic insights from electron and ion microprobe analysis of olivine and orthopyroxene. Geochim. Cosmochim. Acta 1995, 59, 3061-3070. [CrossRef]

30. Lehner, W.; Petaev, M.I.; Buseck, P.R. Formation of niningerite by silicate sulfidation in EH3 enstatite chondrites. Geochim. Cosmochim. Acta 2013, 101, 34-56. [CrossRef]

31. Ballhaus, C.G.; Stumpfl, E.F. Sulfide and platinum mineralization in the Merensky Reef: Evidence from hydrous silicates and fluid inclusions. Contrib. Mineral. Petrol. 1986, 94, 193-204. [CrossRef]

32. Smith, E.M.; Shirey, S.B.; Nestola, F.; Bullock, E.S.; Wang, J.; Richardson, S.H.; Wang, W. Large gem diamonds from metallic liquid in Earth's deep mantle. Science 2016, 354, 1403-1405. [CrossRef] [PubMed]

33. Kullerud, G.; Yoder, H.S., Jr. Sulfide-Silicate Relations: Carnegie Institution of Washington Year Book; Carnegie Institution of Washington: Washington, DC, USA, 1963; Volume 62, pp. 215-218.

34. Los, C.; Bach, W. Sulfidation of major rock types of the oceanic lithosphere; An experimental study at $250{ }^{\circ} \mathrm{C}$ and 400 bars. Lithos 2018, 323, 208-217. [CrossRef]

35. Bataleva, Y.V.; Palyanov, Y.N.; Borzdov, Y.M.; Sobolev, N.V. Sulfidation of silicate mantle by reduced S-bearing metasomatic fluids and melts. Geology 2016, 44, 271-274. [CrossRef]

36. Zhang, Z.; von der Handt, A.; Hirschmann, M. An experimental study of Fe-Ni exchange between sulfide melt and olivine at upper mantle conditions: Implications for mantle sulfide compositions and phase equilibria. Contrib. Mineral. Petrol. 2018, 173, 19. [CrossRef]

37. Bataleva, Y.V.; Palyanov, Y.N.; Borzdov, Y.M. Sulfide formation as a result of sulfate subduction into silicate mantle (experimental modeling under high P,T-parameters). Minerals 2018, 8, 373. [CrossRef]

38. Bataleva, Y.V.; Palyanov, Y.N.; Borzdov, Y.M.; Zdrokov, E.V.; Sobolev, N.V. Experimental modeling of the interaction of subducted carbonates and sulfur with mantle silicates. Dokl. Earth Sci. 2016, 470, 953-956. [CrossRef]

39. Palyanov, Y.N.; Borzdov, Y.M.; Khokhryakov, A.F.; Kupriyanov, I.N.; Sokol, A.G. Effect of nitrogen impurity on diamond crystal growth processes. Cryst. Growth Des. 2010, 10, 3169-3175. [CrossRef]

40. Pal'yanov, Y.N.; Sokol, A.G.; Borzdov, Y.M.; Khokhryakov, A.F. Fluid-bearing alkaline carbonate melts as the medium for the formation of diamonds in the Earth's mantle: An experimental study. Lithos 2002, 60, 145-159. [CrossRef]

41. Palyanov, Y.N.; Sokol, A.G. The effect of composition of mantle fluids/melts on diamond formation processes. Lithos 2009, 112, 690-700. [CrossRef]

42. Sokol, A.G.; Borzdov, Y.M.; Palyanov, Y.N.; Khokhryakov, A.F. High-temperature calibration of a multi-anvil high pressure apparatus. High Press. Res. 2015, 35, 139-147. [CrossRef]

43. Brazhkin, V.V.; Popova, S.V.; Voloshin, R.N. Pressure-temperature phase diagram of molten elements: Selenium, sulfur and iodine. Phys. B Condens. Matter 1999, 265, 64-71. [CrossRef]

44. Sharp, W.E. Melting curves of sphalerite, galena, and pyrrhotite and the decomposition curve of pyrite between 30 and 65 kilobars. J. Geophys. Res. 1969, 74, 1645-1652. [CrossRef]

45. Boehler, R. Melting of the Fe-FeO and the Fe-FeS systems at high pressure-Constraints on core temperatures. Earth Planet. Sci. Lett. 1992, 111, 217-227. [CrossRef]

46. Zhang, Z.; Lentsch, N.; Hirschmann, M.M. Carbon-saturated monosulfide melting in the shallow mantle: Solubility and effect on solidus. Contrib. Mineral. Petrol. 2015, 170, 47. [CrossRef]

47. Zhang, Z.; Hirschmann, M.M. Experimental constraints on mantle sulfide melting up to 8 GPa. Am. Mineral. 2016, 101, 181-192. [CrossRef]

48. Iishi, K. Lattice dynamics of forsterite. Am. Mineral. 1978, 63, 1198-1208. 
49. Guyot, F.; Boyer, H.; Madon, M.; Velde, B.; Poirier, J.P. Comparison of the Raman microprobe spectra of $(\mathrm{Mg}, \mathrm{Fe})_{\mathrm{z}} \mathrm{SiO}_{4}$ and $\mathrm{Mg}_{\mathrm{z}} \mathrm{SiO}_{4}$ with olivine and spinel structure. Phys. Chem. Miner. 1986, 13, 91-95. [CrossRef]

50. Price, G.D.; Parker, S.C.; Leslie, M. Lattice dynamics of forsterite. Mineral. Mag. 1986, 51, 157-170. [CrossRef]

51. Kolesov, B.A.; Geiger, C.A. A Raman spectroscopic study of Fe-Mg olivines. Phys. Chem. Miner. 2004, 31, 142-154. [CrossRef]

52. Huang, E.; Chen, C.H.; Huang, T.; Lin, E.H.; Xu, J.-A. Raman spectroscopic characteristics of Mg-Fe-Ca pyroxenes. Am. Miner. 2000, 85, 473-479. [CrossRef]

53. Gillet, P.; Biellmann, C.; Reynard, B.; McMillan, P. Raman Spectroscopic Studies of Carbonates Part I: High-Pressure and High-Temperature Behaviour of Calcite, Magnesite, Dolomite and Aragonite. Phys. Chem. Miner. 1993, 20, 1-18. [CrossRef]

54. Buzgar, N.; Apopei, A.I. The Raman study on certain carbonates. Analele Ştiinţifice Ale Universităţii "AL. I. CUZA" IAŞI 2009, 55, 97-112.

55. Gunn, S.C.; Luth, R.W. Carbonate reduction by Fe-S-O melts at high pressure and high temperature. Am. Mineral. 2006, 91, 1110-1116. [CrossRef]

56. Bataleva, Y.V.; Palyanov, Y.N.; Borzdov, Y.M.; Kupriyanov, I.N.; Sokol, A.G. Synthesis of diamonds with mineral, fluid and melt inclusions. Lithos 2016, 265, 292-303. [CrossRef]

57. Fleet, M.E.; MacRae, N.D. Partition of Ni between olivine and sulfide and its application to Ni-Cu sulfide deposits. Contrib. Mineral. Petrol. 1983, 83, 75-81. [CrossRef]

58. Heymann, D. Raman study of olivines in 37 heavily and moderately shocked ordinary chondrites. Geochim. Cosmochim. Acta 1990, 54, 2507-2510. [CrossRef]

59. Michel, R.; Ammar, M.R.; Poiriern, J.; Simon, P. Phase transformation characterization of olivine subjected to high temperature in air. Ceram. Int. 2013, 39, 5287-5294. [CrossRef]

60. Weber, I.; Böttger, U.; Pavlov, S.G.; Jessberger, E.K.; Hübers, H.-W. Mineralogical and Raman spectroscopy studies of natural olivines exposed to different planetary environments. Planet. Space Sci. 2014, 104, 163-172. [CrossRef]

61. Wickham-Eade, J.E.; Burchell, M.J.; Price, M.C.; Hicks, L.J.; MacArthur, J.L.; Bridges, J.C. Raman identification of olivine grains in fine grained mineral assemblages fired into aerogel. Proc. Inst. Mech. Eng. 2017, 204, 413-420. [CrossRef]

62. Crepisson, C.; Blanchard, M.; Lazzeri, M.; Balan, E.; Sanloup, C. New constraints on Xe incorporation mechanisms in olivine from first-principles calculations. Geochim. Cosmochim. Acta 2018, 222, 146-155. [CrossRef]

63. Park, S.Y.; Park, C. Spatially-resolved mineral identification and depth profiling on chondrules from the primitive chondrite Elephant Moraine 14017 with confocal Raman spectroscopy. Spectrochim. Acta Part A Mol. Biomol. Spectrosc. 2019, 207, 46-53. [CrossRef] [PubMed]

64. Solovova, I.P.; Kogarko, L.N.; Averin, A.A. Conditions of sulfide formation in the metasomatized mantle beneath East Antarctica. Petrology 2015, 23, 519-542. [CrossRef]

65. Mungall, J.E.; Su, S. Interfacial tension between magmatic sulfide and silicate liquids: Constraints on kinetics of sulfide liquation and sulfide migration through silicate rocks. Earth Planet. Sci. Lett. 2005, 234, 135-140. [CrossRef]

66. Griffin, W.L.; Graham, S.; O’Reilly, S.Y.; Pearson, N.J. Lithosphere evolution beneath the Kaapvaal Craton: Re-Os systematics of sulfides in mantle-derived peridotites. Chem. Geol. 2004, 208, 89-118. [CrossRef]

(C) 2019 by the authors. Licensee MDPI, Basel, Switzerland. This article is an open access article distributed under the terms and conditions of the Creative Commons Attribution (CC BY) license (http://creativecommons.org/licenses/by/4.0/). 